(C) 2000 International Press

Adv. Theor. Math. Phys. 3 (2000) 727-773

\title{
F-Theory with Quantized
}

\section{Fluxes}

Michael Bershadsky ${ }^{1}$, Tony Pantev $^{2}$, and Vladimir Sadov ${ }^{1}$

${ }^{1}$ Lyman Laboratory of Physics

Harvard University

Cambridge, MA 02138

${ }^{2}$ Department of Mathematics

University of Pennsylvania

Philadelphia, PA 19104

\begin{abstract}
We present evidence that the CHL string in eight dimensions is dual to F-theory compactified on elliptic $\mathrm{K} 3$ with a $\Gamma_{0}(2)$ monodromy group. The monodromy group $\Gamma_{0}(2)$ allows one to turn on the flux of an antisymmetric two form along the base. The $B_{\mu \nu}$ flux is quantized and therefore the moduli space of the CHL string is disconnected from the moduli space of Ftheory/Heterotic strings (as expected). The non-zero $B_{\mu \nu}$ flux obstructs certain deformations restricting the moduli of elliptic K3 to a 10 dimensional moduli space. We also discuss how one can reconstruct the gauge groups from the elliptic fibration structure.
\end{abstract}

e-print archive: http://xxx.lanl.gov/hep-th/9805056 


\section{Introduction}

F-theory appears to be a very powerful tool in analyzing the non perturbative aspects of the heterotic string compactifications [20]. The $E_{8} \times E_{8}$ and the $S O(32)$ heterotic string theories are equivalent to each other below ten dimensions. By now we have a very clear understanding of the relation between heterotic strings and F-theory compactifications.

Another interesting construction is the CHL string which exists only for $d \leq 9$ [8], [26]. The CHL string is defined in 9 dimensions as a certain orbifoldization of $E_{8} \times E_{8}$ heterotic string. The orbifold group permutes the two $E_{8}$ 's and acts on $S^{1}$ by a shift. In 8 dimensions one can also discuss CHL either as a $\operatorname{Spin}(32) /(\mathbf{Z} / 2)$ heterotic string with no zero $\mathbf{Z} / 2$ flux. This $\mathbf{Z} / 2$ flux according to Witten [27] measures the obstruction to "vector structure".

One can also discuss the CHL string as a $E_{8} \times E_{8}$ heterotic string with nontrivial bundle twisted by an external $\mathbf{Z} / 2$ automorphism exchanging two $E_{8}$ [27], [19]. In contrast with the heterotic string the CHL strings may have unbroken non-simply laced gauge groups below 9 dimensions. Some aspects of 8 dimensional compactifications were considered in [27] where in particular the dual F-theory is investigated in the isotrivial limit.

The dual description of CHL string in 6 dimensions and below in terms of type II strings was developed by A. Sen and J. Schwarz in [26]. In the second section we review their construction from a slightly different angle. It appears that the construction of [26] can be pushed up to 7 dimensions but the 8 dimensional generalization is not so straightforward.

In the third section we discuss our proposal for a F-theory dual to the CHL string (in 8 dimensions). We claim that the CHL string is dual to a F-theory on an elliptic K3 with non-zero flux of an antisymmetric 2-form $B$ through the sphere. The value of the flux is quantized and it is fixed to be equal to $\frac{1}{2} \omega^{1}$. The presence of this flux freezes 8 of the moduli of the elliptic K3, leaving only a 10 dimensional subspace. On this subspace the monodromy group reduces from $S L(2, \mathbf{Z})$

\footnotetext{
${ }^{1}$ Only the cohomology class of the antisymmetric 2 -form $B_{\mu \nu}$ has a physical meaning. Here $\omega$ denotes the Kahler class of the sphere.
} 
to $\Gamma_{0}(2)$. This is the biggest subgroup of $S L(2, \mathbf{Z})$ that keeps the flux $B=\frac{1}{2} \omega$ invariant. We will check our conjecture by comparing the gauge groups appearing in the F-theory and CHL string compactifications. The possibility of introducing a quantized $B_{\mu \nu}$ flux for certain type I backgrounds was already discussed in [7], [6]. Following Sen [25] one should expect a close relation between F-theory vacua and some type I string backgrounds.

The mechanism of restricting the moduli to a certain sub-locus appears to be very interesting. For example, we can turn on a flux $B=\frac{k}{3} \omega$ (with $k=1,2$ ), restricting the moduli space of elliptic K3 to $\Gamma_{0}(3)$ subloci (in this case the moduli space turns out to have dimension six). The other interesting possibilities are $\Gamma_{0}(4)$ or $\Gamma_{0}(6)$ monodromy groups.

There is another interesting direction that is worth exploring - the relation with an $\mathrm{M}$-theory compactified on a non-commutative torus [9], [13]. Our proposal that the CHL string is dual to a F-theory with $B_{\mu \nu}$ flux through $P^{1}$ seems to be closely related to the setup considered in [13]. According to [13] the positions of the 7-branes cease to commute in the presence of a $B_{\mu \nu}$ background. It is possible that various puzzles raised in this paper can be resolved by using non-commutative geometry.

\section{Low dimensional cases: $7 \mathrm{~d}, 6 \mathrm{~d}$}

Let us start first with a six dimensional compactification $(d=3)$ (for reveiw of low dimensional compactifications see [17] and references therein). A simple generalization of the heterotic-type II duality implies that the CHL string is dual to the M theory on

$$
X_{\sigma}=\left(K 3 \times S^{1}\right) /(\sigma \times(-1)),
$$

where $\sigma$ is an involution acting with eight fixed points on $K 3$ and -1 is the half period shift on $S^{1}$. The action of $\sigma \times(-1)$ has no fixed points on $K 3 \times S^{1}$ and therefore the quotient $X_{\sigma}$ is smooth and can serve as a good gravitational background [26]. The number of deformations of $\mathrm{K} 3$ invariant with respect to the involution $\sigma$ is equal to 12 (= number of hypermultiplets in $6 \mathrm{~d}$ ). This number can either be counted by using the supergravity technique or just by counting directly the invariant 
part of the K3 cohomology. The moduli space of this compactification coincides with the moduli space of the CHL string compactified on a 3 -dimensional torus and is given by a coset space

$$
\mathcal{M}_{d}=\Gamma \backslash S O(12,4) /(S O(12) \times S O(4)),
$$

where $\Gamma$ is a certain discrete group. The perturbative type IIA description of this compactification is rather obscure because the manifold $X_{\sigma}$ cannot be treated as an $S^{1}$ bundle over the quotient $K 3 / \sigma$ : the radius of the would-be fiber $S^{1}$ is not constant over $K 3 / \sigma$ since it jumps at the orbifold points. In the M-theory approach it is more natural to view $X_{\sigma}$ as a nontrivial $K 3$-bundle over $S^{1}$ so that the limit when the circle gets large can be described using adiabatic arguments. In the latter approach the singular quotient $K 3 / \sigma$ never appears. Still, for our 8-dimensional applications we will need to understand the behavior of various degenerations of this quotient.

At the generic point of the moduli space 2 the gauge symmetry is broken to $U(1)^{16}$. Now let us discuss how the nonabelian gauge groups appear. In this compactification we will see both simply-laced (A-D-E) and non-simply-laced ( $B$ and $C$ ) gauge groups. Recall that a simply laced group appears in M-theory compactified on $K 3$ whenever there is a singularity on $K 3$ resulting from collapsing a collection of 2-cycles intersecting each other according to an A-D-E Dynkin diagram. To find the simply laced gauge groups in M-theory on $X_{\sigma}$ suppose that there is a singular point $P$ on $K 3$ not fixed by the involution $\sigma$, so that $\sigma(P)=P^{\prime}$. The point $P^{\prime}$ has to be singular also with the singularity of $K 3$ locally isomorphic to that at $P$. Considering $X_{\sigma}$ as a bundle over the large radius circle $S^{1}$ we see that each fiber has (at least) two singularities at $P$ and $P^{\prime}$ exchanged by the monodromy around the circle. Using adiabatic arguments the readers can easily convince themselves that the resulting gauge group appearing in the compactification of M-theory on this bundle is simply laced level 2. This can also be recognized as a reformulation in the M-theory context of a statement in PolchinskiChaudhuri [8].

The mechanism responsible for the appearance of non-simply laced gauge groups is different. Let us first note that at special points in the moduli space the involution $\sigma$ has invariant spheres ${ }^{2}$. When such a sphere collapses to a point $K 3$ develops an $A_{1}$ singularity fixed by $\sigma$.

\footnotetext{
${ }^{2}$ The existence of such spheres is a non-trivial condition on $K 3$ which will be
} 
Each such sphere passes through exactly two fixed points. Contraction of any of these spheres leads to an enhanced gauge symmetry $S p(1)=$ $S U(2)$. This construction can be immediately generalized in order to get $S p(n)$ gauge groups. Consider a collection of $2 n+1$ vanishing spheres intersecting according $A_{2 n+1}$ Dynkin diagram. This sublattice $A_{2 n+1} \subset H^{2}(K 3, \mathbf{Z})$ maps on itself under the involution $\sigma$ in such a way that two $A_{n}$ sublattices $\left\{S_{i}\right\}$ and $\left\{S_{n+1+i}\right\}$ (where $i=1, \ldots, n$ ) are permuted

$$
S_{i} \longleftrightarrow S_{2 n+2-i}
$$

keeping the middle sphere $S_{n+1}$ invariant (see Fig.1).

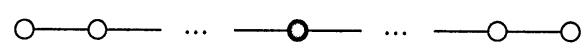

Figure 1:

One can immediately recognize that the involution $\sigma$ acts by an external automorphism of $A_{2 n+1}$ Dynkin diagram. This involution basically folds the Dynkin diagram on itself. The $S p(n)$ gauge symmetry appears after contraction of the diagram.

To make the discussion more concrete we will use a particular family of elliptic $K 3$ with Weierstrass representation given by

$$
y^{2}=x^{3}+x f_{8}(z)+g_{12}(z),
$$

where $f_{8}(z)$ and $g_{12}(z)$ are polynomials on the base satisfying the relation $z^{8} f_{8}(1 / z)=f_{8}(z)$ and $z^{12} g_{12}(1 / z)=g_{12}(z)$. One can immediately verify that 4 is a 10 dimensional family of K3's. The involution $\sigma$ acts on 4 in a very simple way: $y \rightarrow-y$ and $z \rightarrow 1 / z$.

The fixed points of $\sigma$ are located in two fibers projecting to $z= \pm 1$ on the base. The quotient $K 3 / \sigma$ does not have a Weierstrass representation, but it is still an elliptic fibration with two $\hat{D}_{4}$ fibers where the double sphere is the image of the elliptic fiber of $K 3$.

In this model one can easily understand what happens when an invariant sphere for $\sigma$ appears. To obtain this situation let us choose

automatically satisfied for the F-theory compactifications dual to a CHL string. See the discussion in Sections 3, 4 for an explanation. 
$f$ and $g$ such that there is an $I_{2}$ singular fiber at $z=1$. Then on the quotient the fiber is $I_{1}^{*}$. Similarly, whenever the fiber at $z=1$ is $I_{2 n}$ (the index can only be even because of $\mathbf{Z}_{2}$ invariance), the fiber on the quotient is $I_{n}^{*}$. The cycles in $I_{n}^{*}$ represent the affine Dynikin diagram $\hat{D}_{n+4}$ : the four terminal points of the graph represent the four orbifold points, the two cycles immediately adjacent to them are the ones left invariant by $\sigma$ upstairs and the $n-1$ cycles in the middle are images of $2(n-1)$ cycles upstairs (see Fig.2).

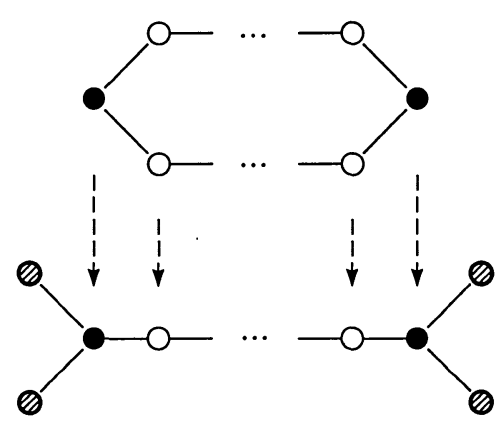

Figure 2: $D_{4+n}$ singularity, four terminal cycles represent the orbifold points.

The cycles upstairs represent the $\hat{A}_{2 n-1}$ affine Dynkin diagram. The involution folds this diagram on itself keeping two opposite cycles fixed and attaches to each invariant cycle two more cycles. The singularity which corresponds to contraction of all these cycles except one is $\left(A_{1}\right)^{4}$ for $n=0, A_{3} \times\left(A_{1}\right)^{2}$ for $n=1$ and $D_{n+2} \times\left(A^{1}\right)^{2}$ for $n>1$.

The following observation will be important for our discussion of 8-dimensional compactifications in the next section. From the point of view of the quotient $K 3 / \sigma$ the above situation looks as if two orbifold points and an $A_{n}$ singularity all collide at one point: notice that $A_{n} \times\left(A_{1}\right)^{2} \subset D_{n+2}$, where the factor $\left(A_{1}\right)^{2}$ describes the orbifold singularities. To summarize, the orbifold points do not lead to massless vector particles and in spite of the fact that the singularity on the quotient $K 3 / \sigma$ is $D_{n+2} \times\left(A_{1}\right)^{2}$ the gauge group is $S p(n)$.

In a similar fashion one can also explain the appearance of $S O(2 n+$ 1) level 1 . Consider the elliptic K3 with a degenerate fiber $I_{4}$ located at $z=1$ and a degenerate fiber $I_{2}$ located at $z=-1$. The zero section, 
which is invariant under $\sigma$ intersects one invariant sphere in both fibers (see Fig. 3).

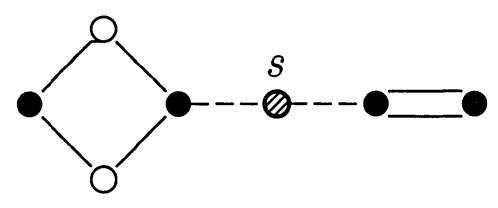

Figure 3: The sphere marked by $S$ represents a zero section.

In turn, the invariant sphere in $I_{4}$ intersects two more spheres, which are permuted by $\sigma$. All in all, there are five cycles representing a $D_{5}$ Dynkin diagram. The involution maps the diagram on itself resulting in $B_{4}$ diagram. Contraction of all these spheres yields $B_{4}=S O(9)$ gauge group. In order to get $S O(7)$ one has to start with $I_{4}$ singularity located either at $z=1$ or $z=-1 . S O(9)$ is the maximal $S O(2 n+1)$ gauge group that can appear in 7 dimensions. In 6 dimensions the maximal $S O(2 n+1)$ gauge group is $S O(13)$. To see it one needs to adjust the radius of the circle $S^{1}$.

To obtain compactifications dual to CHL string in $7 \mathrm{~d}$ rather than in 6d we may use a trick due to Aspinwall and Schwarz [1]. We assume that K3 is elliptic and consider the limit in which the Kähler class of the elliptic fiber tends to zero. This is the limit in which one space dimension gets decompactified, as described in [1] in detail. The resulting $7 \mathrm{~d}$ theory can be described as a F-theory compactification on the quotient space $\left(K 3 \times S^{1}\right) /(\mathbf{Z} / 2)$. The other way to obtain this result is to use the duality between F-theory on $K 3$ and heterotic string on $T^{2}$ in 8 dimensions and compactify both theories on $S^{1}$ with PolchinskyChaudhuri twist. The discussion of gauge groups appearing in this compactification remains the same as in the six-dimensional case.

\section{$3 \quad$ F-theory with fluxes}

In this section we describe the F-theory compactification (in 8 dimensions) dual to the CHL string. Such F-theory compactifications were analyzed by Witten in [27] in the limiting locus when the elliptic fi- 
bration becomes isotrivial (this limit was also discussed in [6], [24]). We extend Witten's description to the interior of the moduli space and confirm that even though the F-theory elliptic fibration is no longer isotrivial the essential features of Witten's description are retained. The extra structure of the compactification exploited here is dictated to us by the specialization of the usual Heterotic/F-theory duality to the CHL case. This transition is explained in detail in the next section.

We will present strong evidence showing that CHL is dual to Ftheory compactified on elliptic K3 with non-zero flux of an antisymmetric field $B_{\mu \nu}$ along the base sphere. According to the conventional wisdom, the single valued background of antisymmetric 2-forms (NSNS and RR) contradicts the $S L(2, \mathrm{Z})$ monodromy. Luckily, there is a way out of this contradiction when the monodromy group is smaller than $S L(2, \mathbf{Z})$. The K3 surface on which our F-theory is compactified moves in a 10 dimensional family of elliptic fibrations with monodromy contained in the congruence subgroup $\Gamma_{0}(2)$. This monodromy group does allow (see Section 4.4 for more details) a non-zero value of the antisymmetric 2 -form. Before we proceed let us make some comments about the $B_{\mu \nu}$ backgrounds. The antisymmetric two form is defined modulo gauge transformations $B \rightarrow B+d \Lambda$. Therefore, turning the $B_{\mu \nu}$ background along the $\mathbf{P}^{1}$ (the base of F-theory compactification) introduces only two new real parameters - the classes of $B^{N S}=b \omega$ and $B^{R R}=b^{\prime} \omega$. Both $b$ and $b^{\prime}$ take values in $S^{1}$. Moreover, the field strength $H=d B$ is identically equal to zero for this background and therefore, locally one can gauge away the $B_{\mu \nu}$ field. This will become important in our further discussion.

The monodromy group $\Gamma_{0}(2)$ keeps one of the three half-periods invariant. For example, realizing $\Gamma_{0}(2)$ by $S L(2, \mathbf{Z})$ matrices with even entries in the lower left corner we get

$$
\left(\begin{array}{cc}
a & b \\
2 k & d
\end{array}\right)\left(\begin{array}{c}
1 / 2 \\
0
\end{array}\right)=\left(\begin{array}{c}
a / 2 \\
k
\end{array}\right) \equiv\left(\begin{array}{c}
1 / 2 \\
0
\end{array}\right)(\bmod \mathbf{Z})
$$

This is the reason why one can turn on an antisymmetric 2-form without ruining the F-theory structure, by setting $b=1 / 2$ and $b^{\prime}=0$. Moreover, the non-zero $B_{\mu \nu}$ background obstructs certain deformations of the elliptic K3, allowing only those compatible with $\Gamma_{0}(2)$ monodromy. 
The formulation of F-theory as type IIB string is sensitive only to the complex moduli of elliptic manifold and it does not care about the Kähler moduli. Therefore before going further we have to choose an appropriate model for elliptic K3 with $\Gamma_{0}(2)$ monodromy. Let us first describe a $\mathrm{K} 3$ which is the Weierstrass model of an elliptic fibration with $\Gamma_{0}(2)$ monodromy. $\Gamma_{0}(2)$ preserves one of the non-trivial spin structures (half-periods) of the fiber. That means that besides the always present section at infinity, the elliptic fibration has another global section given by that half-period. The appropriate Weierstrass form can be written as

$$
y^{2}=\left(x-a_{4}(z)\right)\left(x^{2}+x a_{4}(z)+b_{8}(z)\right),
$$

where $a_{4}(z), b_{8}(z)$ are two polynomials of degree 4 and 8 on the base of the elliptic fibration. It is the reducibility of the right hand side of 6 that ensures the existence of the global section $y=0, x=a_{4}$. The Weierstrass equation 6 describes a 10 parameter family of elliptic K3 surfaces. The minimal resolution of all K3's in this family have eight singular fibers of Kodaira type $I_{2}$ as can be easily seen from the discriminant

$$
\Delta=\left(b_{8}+2 a_{4}^{2}\right)^{2}\left(4 b_{8}-a_{4}^{2}\right)
$$

The zeroes of $\left(b_{8}+2 a_{4}^{2}\right)$ determine the locations of eight $I_{2}$ fibers. At the generic point in the moduli space such a K3 has eight $A_{1}$ singularities localized on the extra section $y=0, x=a_{4}$.

It turns out however that the moduli space of F-theory compactifications corresponding to the CHL string is a finite cover of the moduli space of $\Gamma_{0}(2)$ Weierstrass models just described. Concretely in order to recover the dual CHL compactification from the Weierstrass model 6 one also needs to choose 4 of the eight points on the base at which the $I_{2}$ fibers are located (see Section 4.4 for an explanation). With this extra data the elliptic curve on which the dual CHL string is compactified is recovered as the double cover of $\mathbf{P}^{1}$ branched at the 4 marked points and the twisted $E_{8} \times E_{8}$ instanton of the CHL compactification is encoded in the complex structure of the surface 6 . This situation is very different from the standard F-theory/Heterotic correspondence. In the later case the elliptic curve of Heterotic compactification (at least in the vicinity of $E_{8} \times E_{8}$ locus) was encoded in the elliptic fiber. 
Actually from the duality with the CHL string one canonically reconstructs (see Sections 4.3 and 4.4) a slightly different birational model of 6 obtained as follows. The minimal resolution of 6 is a smooth elliptic K3 having eight $I_{2}$ fibers and two sections arranged in such a way that at each $I_{2}$ fiber the two sections pass trough two different components of the fiber. If we now contract the four components of 6 meeting the section $y=0, x=a_{4}$ at the four marked points on the base and contract the components meeting the section at infinity at the remaining 4 points we will get an elliptic $\mathrm{K} 3$ which also has eight $A_{1}$ singularities and is the same as 6 outside of the $I_{2}$ fibers. In particular this new K3 has the same $j$-function and the same monodromy as 6 but is not in Weierstrass form since each of the two sections now passes trough four of the singular points.

To make a connection between this picture (in 8 dimensions) and the one given above in lower dimensions let us further compactify Ftheory on an extra $T^{2}$. As the result we get a 6 -dimensional theory which can be interpreted as M-theory on $K 3 \times S^{1} / \sigma$, described in the previous section. Let us denote the quotient $K 3 / \sigma$ by $X$. As we already explained in the previous section $X$ is a singular K3 surface. The surface $X$ turns out to be quite remarkable. It admits several elliptic fibrations two of them being particularly important. One elliptic fibration is inherited from the $K 3$ upstairs (the Weierstras model for the $K 3$ upstairs is given by 6) and will be called the inherited elliptic fibration. In another elliptic fibration, to be called the $\Gamma_{0}(2)$ elliptic fibration, the monodromy group is $\Gamma_{0}(2)$ and there are two sections (the zero section and the section at infinity) with four $A_{1}$ singularities on top of each. Thus the surface $X$ is exactly the elliptic K3 we chose to compactify F-theory on.

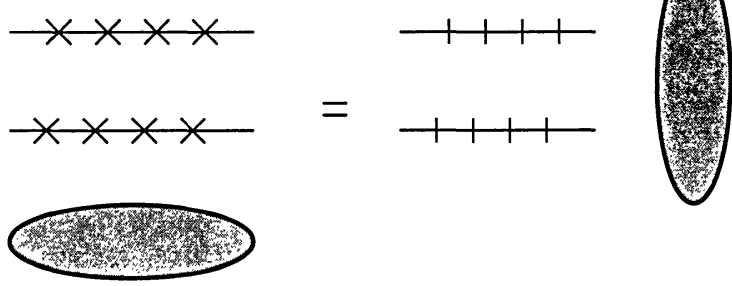

Figure 4: Two elliptic fibrations. 
The existence of two elliptic structures simplifies significantly the identification of the points on the moduli space corresponding to nonabelian gauge groups. Particularly useful is the following simple remark. Two sections with four $A_{1}$ singularities in the fibration with $\Gamma_{0}(2)$ are the fibers over the fixed points $(z= \pm$ in 4$)$ in the another fibration.

Now we can turn to the discussion of gauge groups in the F-theory language. The CHL string has A-D-E level 2 as well as $C_{n}=S p(n)$ level 1 gauge groups ${ }^{3}$. In the $\mathrm{F}$-theory without the flux the gauge groups would appear at points in the moduli space where elliptic fibration develops a singularity in the elliptic fiber. In the presence of fluxes the situation should becomes more involved. For example, $E_{6}$ or $E_{8}$ degeneration of the elliptic fiber cannot appear in elliptic K3 with $\Gamma_{0}(2)$ monodromy. Still, we expect that these gauge groups appear in 8-dimensional compactifications of the CHL string. Moreover, we will see that in certain cases the presence of an $I_{2}$ singularity does not necessarily lead to a $S U(2)$ gauge group. It seems unlikely that these phenomena can be explained from the point of view of local D-brane physics in the presence of a $B_{\mu \nu}$ field. Indeed, by choosing an appropriate gauge locally one can put the $B_{\mu \nu}$ field equal to zero getting back the familiar D-brane picture. There is clearly a global obstruction of getting rid of $B_{\mu \nu}$ field everywhere and therefore the absence of the enhanced gauge symmetry one naively expects is forced by the global topological properties of the compactification ${ }^{4}$. As explained later, geometrically this is expressed in the relative position of the additional section and the singular fiber responsible for the appearance of the gauge symmetry.

We suggest the following "strategy" to derive the gauge groups. As we have seen the K3 surface $X$ has multiple personalities and disguises either as a space on which some F-theory with fluxes is compactified or as an object dual to the CHL string. This personality disorder is encoded in the existence of the inherited and the $\Gamma_{0}(2)$-elliptic fibrations on $X$ and so the idea is to compare the singularities that appear in these two elliptic fibrations. Clearly, a fiber singular in one of the

\footnotetext{
${ }^{3}$ The maximal $S O(2 n+1)$ gauge group that appear in 8 dimensions is $S O(5)=$ $S p(2)$.

${ }^{4}$ Note that in general the global topology does not kill the observed gauge group completely but always reduces the naive gauge symmetry to a smaller one.
} 
fibrations does not necessarily correspond to a singular fiber in the other elliptic fibration. It may correspond to a collection of singular fibers connected by a section (or by sections). For simple gauge groups the corresponding singularities of the total space after the resolution can be localized on one singular fibers in each elliptic fibration. By the nature of the duality construction the CHL gauge groups can be detected by analyzing the singular fibers in the inherited elliptic fibration. For all gauge groups but the exceptional ones, the Dynkin diagram of the gauge group coincides with the common part of the singular fibers taken in both elliptic fibrations, with the cycles corresponding to the fixed points being dropped out. Therefore to see these groups in the Ftheory with fluxes we need to translate (see Section 4.5) the singularities into collision patterns for the $\Gamma_{0}(2)$-fibration. This can be done either directly on $X$ or on its $\Gamma_{0}(2)$-Weierstrass model $w: Y \rightarrow \mathbf{P}^{1}$. In the latter case however we need to remember the extra data of splitting the eight $I_{2}$ fibers into two groups of four $I_{2}$ fibers each.

For example, if a Kodaira fiber of affine A-D-E type appears in the inherited elliptic fibration in a way that does not involve collision with the two $\hat{D}_{4}$ fibers, then when viewed in the $\Gamma_{0}(2)$-elliptic fibration this fiber gives rise to the condition for the appearance of an A-D-E gauge group of level 2. In order to get the $S p(n)$ level 1 gauge groups one has to start with $I_{n}$ colliding with one of the $\hat{D}_{4}$ fibers. As the result we get the following "dictionary".

\section{$3.1 S p(n)$ gauge groups}

The $S p(n)$ gauge groups appear as the result of collision of two $I_{2}$ fibers from the same section and $n I_{1}$ fibers for $n>1$. In terms of the Weierstrass model $Y$ this just means that the two colliding $I_{2}$ fibers belong to the same group of four.

The case $S p(1)=S U(2)$ is special and this gauge group appears when just two $I_{2}$ fibers collide with each other. For every $n>1$ the collision $2 I_{2}+n I_{1}$ produces a $\hat{D}_{n+2}$ singular fiber in the $\Gamma_{0}(2)$-fibration $\hat{\pi}: \hat{X} \rightarrow \mathbf{P}^{1}$ on the minimal resolution of $\hat{X}$. The corresponding singularity of $X$ (or of the Weierstrass model $Y$ ) is obtained by all cycles in $\hat{D}_{n+2}$ which do not meet the zero section of $Y$. This cycles form an ordinary $D_{n+2}$ Dynkin diagram and the two cycles on the tail 
of this $D_{n+2}$ correspond to two of the branch points of the covering $S \rightarrow X$. The other $n$ represent the roots of $S p(n)$. The maximal rank that can be obtained this way is 8 . Still, in CHL string one can get get $S p(9)$ and $S p(10)$. In this case the monodromy group further reduces to $\Gamma(2) \in \Gamma_{0}(2)$.

\section{$3.2 \quad S U(n)$}

Collision of $n$ fibers of type $I_{1}$ among themselves leads to a $S U(n)$ gauge group. The maximal rank that can be obtained in this way is 8 .

\section{3 $S O(2 n)$ gauge groups}

The $S O(2 n)$ gauge groups appear as the result of collision of two $I_{2}$ fibers from different sections and $n I_{1}$ fibers for $n>1$. In the Weiersrass model this just corresponds to the collision of two $I_{2}$ fibers belonging to two different groups of four. The maximal gauge group that appears this way is $S O(16)$.

\subsection{Exceptional gauge groups $\left(E_{6}, E_{7}, E_{8}\right)$}

The exceptional groups of the $E_{n}$ series appear when a number of $I_{1}$ fibers in the $\Gamma_{0}(2)$-fibration collide in such a way that the monodromy reduces to the subgroup $\Gamma(2) \subset \Gamma_{0}(2)$ which preserves all points of order two ${ }^{5}$. Concretely this means that the bisection $C \subset X$ splits into two sections. In the Weierstrass model $Y$ given by 6 the curve $C$ is given by the equation $y=0=x^{a}+a_{4}(z) x+b_{8}(z)$ and so the $\Gamma(2)$ degeneration occurs exactly when $x^{2}+a_{4}(z) x+b_{8}(z)$ decomposes as $x^{2}+a_{4}(z) x+b_{8}(z)=(x-p(z))(x-q(z))$ with $p(z), q(z)$ - general polynomials of degree four on $\mathbf{P}^{1}$.

After tracing (see Section 4.5 for details) the correspondence between the singular fiber in the two elliptic fibrations one sees that the exceptional gauge groups appear as follows:

$$
{ }^{5} \text { Explicitly } \Gamma(2)=\left\{\begin{array}{ll}
a & b \\
c & d
\end{array} \in S L(2, \mathbf{Z}) \mid a, d-\text { odd, } b, c-\text { even }\right\}
$$


$\underline{E_{6}}$ : Corresponds to a $\Gamma(2)$-collision of six fibers of type $I_{1}$ in the $\Gamma_{0}(2)$ elliptic fibration.

$E_{7}$ : Can be obtained in two ways. Either as a $\Gamma(2)$-collision of eight fibers of type $I_{1}$ in the $\Gamma_{0}(2)$ elliptic fibration or as a $\Gamma(2)$-collision of six $I_{1}$ fibers with two $I_{2}$ fibers coming from the two different sections in the $\Gamma_{0}(2)$ fibration.

$\underline{E_{8}}$ : Corresponds to a $\Gamma(2)$-collision of eight $I_{1}$ fibers with two $I_{2}$ fibers coming from the two different sections in the $\Gamma_{0}(2)$ fibration.

Furthermore, it turns out (see Section 4.5) that the two mechanisms of $E_{7}$ creation described above are not deformable to each within the locus of deformations of $X$ preserving the $\Gamma(2)$ monodromy. On the other hand the mechanism of crating $E_{6}$ deforms further to $E_{7}$ coming from a $\Gamma(2)$-collision of $8 I_{1}$ and the occurrence of $E_{7}$ as a $\Gamma(2)$-collision of $6 I_{1}+2 I_{2}$ admits a further $\Gamma(2)$ degeneration to the creation of $E_{8}$.

The two ways of creating $E_{7}$ can be deformed to each other by keeping both an $E_{7}$ fiber in the inherited elliptic fibration and a $\Gamma_{0}(2)$ monodromy. The intermediate $\Gamma_{0}(2)$ surfaces however are singular in codimension one and for them the $j$-invariant of the $\Gamma_{0}(2)$-fibration becomes identically $\infty$.

\subsection{Other collisions}

Other collisions usually correspond to products of gauge groups. For example the singularity $3 I_{2}+n I_{1}$ gives rise to the $S p(n) \times S p(1)$ gauge groups for $n \geq 2$ and $(S p(1))^{2}$ gauge group for $n=0$. The series $4 I_{2}+n I_{1}$ corresponds to $S p(n) \times(S p(1))^{2}$ for $n \geq 2$ and $(S p(1))^{3}$ for $n=0$.

Remark. The A-D-C groups of ranks bigger than eight should appear in a way similar to the creation of the exceptional gauge symmetries. In other words one expects higher rank A-D-C's when the monodromy group drops to some finite index subgroup in $\Gamma_{0}(2)$. 


\section{Justification of the CHL/F-theory du- ality}

In this section we transplant the usual duality mechanism relating $\mathrm{F}$ theory and the Heterotic string to the situation of the CHL/F-theory correspondence. For the convenience of the reader and to set up notation the relevant parts of the Heterotic/F-theory duality (see [21], [5], [14] and [12] for more details) are reviewed in the Appendix.

\subsection{The on-the-nose correspondence}

The CHL string can be obtained from the $E_{8} \times E_{8}$ Heterotic string by a certain $\mathbf{Z} / 2$ quotient. The CHL involution acts by a half period shift on the torus $T^{2}$ and permutes the two $E_{8}$. Clearly this involution acts trivially on $\tau$ and $\rho$ (complex and Kähler strictures of $T^{2}$ ) and so the moduli space of vacua for CHL compactified on $T^{2}$ can be naturally identified [8] with the locally symmetric space

$$
O(2,10 ; \mathbf{Z}) \backslash O(2,10 ; \mathbf{R}) /(O(2 ; \mathbf{R}) \times O(10 ; \mathbf{R})) .
$$

Intrinsically the relation of 8 with (5.1) can be described as follows. First use the Narain construction to write (5.1) in a more precise form, namely as the double coset space

$$
O(\mathbf{N a r}) \backslash O(\mathbf{N a r} \otimes \mathbf{R}) / K
$$

where $^{6}$ Nar $:=\mathbf{U} \oplus \mathbf{U} \oplus \mathbf{E}_{8} \oplus \mathbf{E}_{8}$ is the Narain lattice and $K \subset$ $O(\mathbf{N a r} \otimes \mathbf{R})$ is a maximal compact subgroup. As usual the $\mathbf{U} \oplus \mathbf{U}$ part of the Narain lattice is identified ${ }^{7}$ with $H^{2}\left(T^{2}, \mathbf{Z}\right) \oplus H^{2}\left(T^{2}, \mathbf{Z}\right)$. The CHL involution which acts as a translation by a half period on the torus and is homotopic to the identity and so induces the identity on cohomology. Thus the CHL involution $i: \mathrm{Nar} \rightarrow \mathrm{Nar}$ is given explicitly by $i\left(u_{1}, u_{2} ; \xi^{\prime}, \xi^{\prime \prime}\right)=\left(u_{1}, u_{2} ; \xi^{\prime \prime}, \xi^{\prime}\right)$. From that viewpoint the space 8 is naturally written as $O\left(\mathbf{N a r}^{\langle i\rangle}\right) \backslash O\left((\mathbf{N a r} \otimes \mathbf{R})^{\langle i\rangle}\right) /($ maximal compact)

\footnotetext{
${ }^{6}$ Here $\mathbf{U}$ denotes the two dimensional hyperbolic lattice and $\mathbf{E}_{8}$ denotes the root lattice of the group $E_{8}$.

${ }^{7}$ Since the pair $(\tau, \rho)$ intrinsically lives in $\left(H^{2}\left(T^{2}, \mathbf{Z}\right) \oplus H^{2}\left(T^{2}, \mathbf{Z}\right)\right) \otimes \mathbf{R}$
} 
with $\mathrm{Nar}^{\langle i\rangle}$ being the sublattice of $i$-invariants. From the exact form of $i$ one easily sees that ${ }^{8} \mathbf{N a r}^{\langle i\rangle} \cong \mathbf{U} \oplus \mathbf{U} \oplus \mathbf{E}_{8}(2)$ which suggests that the dual F-theory should be compactified on a K3 surface ${ }^{9}$ whose transcendental lattice is at least isogenous to $\mathbf{U} \oplus \mathbf{U} \oplus \mathbf{E}_{8}(2)$. In fact there is a well understood (see [11], Example 9.1 and [22]) moduli space of lattice polarized K3's with this property - the moduli space of K3 surfaces which are universal covers of Enriques surfaces.

Recall [10] that an Enriques surface is a complex surface $F$ with $H^{0}\left(F, \Omega_{F}^{1}\right)=H^{0}\left(F, \Omega_{F}^{2}\right)=0$ but for which $K_{F}^{\otimes 2}=\left(\Omega_{F}^{2}\right)^{\otimes 2}=\mathcal{O}_{F}$. The unramified double covering $p: S \rightarrow F$ corresponding to the 2torsion line bundle $K_{F}$ has trivial canonical class and no holomorphic one forms and is thus a K3 surface. Let $e: S \rightarrow S$ be the fixed point free involution acting along the fibers of $p$. The second cohomology group $H^{2}(F, \mathbf{Z})$ is isomorphic to $\mathbf{U} \oplus \mathbf{E}_{8}$. The pullback map gives an inclusion $p^{*}: H^{2}(F, \mathbf{Z}) /$ torsion $\hookrightarrow H^{2}(S, \mathbf{Z})$ of free abelian groups but since $p$ is two-sheeted we get an isomorphism of Eucledian lattices $H^{2}(S, \mathbf{Z})^{\langle e\rangle}=$ $p^{*} H^{2}(F, \mathbf{Z}) \cong \mathbf{U}(2) \oplus \mathbf{E}_{8}(2)=: M$. One can check [10] that $\operatorname{Pic}(F)=$ $H^{2}(F, \mathbf{Z})$ and that for the generic $F$ all algebraic cycles on $S$ are pullback from cycles on $F$. In this way $S$ acquires a canonical structure of $\mathbf{U}(2) \oplus \mathbf{E}_{8}(2)$-polarized K3 surface. It can be shown [22] that we can choose a marking $H^{2}(S, \mathbf{Z}) \cong \mathbf{U} \oplus \mathbf{U} \oplus \mathbf{U} \oplus \mathbf{E}_{8} \oplus \mathbf{E}_{8}=: \Lambda$ so that the Enriques involution $e$ acts as $e\left(u_{1}, u_{2}, u_{3} ; \xi^{\prime}, \xi^{\prime \prime}\right)=\left(-u_{1}, u_{3}, u_{2} ; \xi^{\prime \prime}, \xi^{\prime}\right)$. In particular the transcendental lattice of a general $S$ will be isomorphic to $\Lambda^{\operatorname{sign}(\langle e\rangle)}=\mathbf{U} \oplus \mathbf{U}(2) \oplus \mathbf{E}_{8}(2)$ and hence the moduli space of all $S$ 's will be isomorphic ${ }^{10}$ to $O\left(\Gamma_{M}\right) \backslash O\left(\Lambda^{\operatorname{sign}(\langle e\rangle)} \otimes \mathbf{R}\right) /($ maximal compact $)$. Up to isogeny this space is isomorphic to 8 and this identification provides the first approximation to the F-theory-CHL duality.

\subsection{The correspondence in the stable limit}

To reconfirm that the F-theory compactifications described in the previous subsection are indeed dual to the CHL string it is instructive to

\footnotetext{
${ }^{8}$ As usual given an Eucledian lattice $L$ and an integer $m$ we denote by $L(m)$ the lattice isomorphic to $L$ as a free abelian group but whose bilinear pairing is $m$ times the pairing of $L$.

${ }^{9}$ See the Appendix of how moduli spaces of lattice polarized K3 surfaces naturally appear in the context of F-theory.

${ }^{10}$ See the discussion in the Appendix.
} 
examine the above correspondence in the stable limit where the complexified Kähler class of the CHL $T^{2}$ is allowed to become infinitely large.

Similarly to the ordinary F-theory/Heterotic duality (see the Appendix) in this regime the vacua for both the CHL string and the Ftheory can be encoded entirely in algebraic-geometric objects. For the CHL string we have:

(a) An elliptic curve $E$ with a marked origin (= the torus $T^{2}$ taken with the complex structure $\tau$ ).

(b) A point of order two $\alpha \in E$ (so that translation $t_{\alpha}: E \rightarrow E$ by $\alpha$ corresponds to the action of the CHL involution on $\left.T^{2}\right)$. We denote the quotient elliptic curve $E /\left\langle t_{\alpha}\right\rangle$ by $Z$ and the natural projection $E \rightarrow Z$ by $q_{\alpha}$.

(c) An $\alpha$-twisted $E_{8} \times E_{8}$ principal bundle $V \rightarrow Z$.

To explain the notion of an $\alpha$-twisting start by recalling that a principal $G$-bundle on a variety $B$ is a variety $P$ equipped with a locally trivial fibration $f: P \rightarrow B$ and a right action $P \times G \rightarrow P$ such that for every $b \in B$ the action on the fiber $P_{b} \times G \rightarrow P_{b}$ is simply transitive. In exactly the same way we can start not with a group $G$ but with a twisted form of $G$, i.e. a bundle of groups $\mathcal{G} \rightarrow B$ which on small open sets $U \subset B$ is isomorphic to the product of $G \times U$. Now a principal $\mathcal{G}$ bundle (or $\mathcal{G}$-torsors) will be just a variety $P$ equipped with a locally trivial fibration $f: P \rightarrow B$ and an action $P \times \mathcal{G} \rightarrow P$ which is fiberwise simply transitive. In the concrete situation above one considers the bundle of groups $\mathcal{E}_{8}^{\alpha} \rightarrow Z$ defined as the push-forward $q_{\alpha *} \underline{E}_{8}$ of the trivial bundle of groups $\underline{E}_{8}=E \times E_{8}$ on $E$. The fiber of $\mathcal{E}_{8}^{\alpha}$ over a point $z \in Z$ is isomorphic to the product of two copies of the group $E_{8}$ labeled by the two points in the fiber $q_{\alpha}^{-1}(z) \subset E$.

According to our on-the-nose correspondence and the usual F-theory/ Heterotic duality the data (a), (b) and (c) will have to correspond to a stable degeneration of a K3 cover of an Enriques surface. In order to describe this degeneration we will have to view the data (a), (b) and (c) as a data describing an ordinary Heterotic string in 8 dimensions. To do that we have to recast the $\alpha$-twisted principal bundle 
$V$ as an object on $E$. For this we just pull $V$ back via the covering map $q_{\alpha}: E \rightarrow Z$. By definition $q_{\alpha}^{*} V$ will be a principal bundle over $q_{\alpha}^{*} \mathcal{E}_{8}^{\alpha}=\underline{E}_{8} \times \underline{E}_{8}$, i.e. an ordinary $E_{8} \times E_{8}$ bundle on $E$. Furthermore $t_{\alpha}^{*} q_{\alpha}^{*} V \cong q_{\alpha}^{*} V$ by construction and so $q_{\alpha}^{*} V=W \times t_{\alpha}^{*} W$ for some $E_{8^{-}}$ bundle $W$ on $E$. Conversely given an $E_{8}$-bundle $W \rightarrow E$ we can define $V=q_{\alpha *} W=\left(q_{\alpha *}\left(W \times t_{\alpha}^{*} W\right)\right)^{\left\langle t_{\alpha}\right\rangle}$. To summarize: the CHL data (a), (b), (c) on $Z$ is equivalent to the Heterotic string data consisting of $E$, the involution $t_{\alpha}: E \rightarrow E$ and an $E_{8}$ bundle $W$ on $E$. By what we have just said the $E_{8} \times E_{8}$ bundle on $E$ that one gets in this way is just the bundle $W \times t_{\alpha}^{*} W$. Therefore, as explained in the Appendix, the stable degeneration of the K3 corresponding to $E$ and $W \times t_{\alpha}^{*} W$ will have to be a union of two rational elliptic surfaces (with sections) $S^{\prime}$ and $S^{\prime \prime}$ intersecting transversally along a copy of $E$ sitting in each of them as an anticanonical section. The only new feature in this picture is that since the two bundles $W$ and $t_{\alpha}^{*} W$ are isomorphic the two rational elliptic surfaces $S^{\prime}$ and $S^{\prime \prime}$ will have to be isomorphic to the rational elliptic surface $J$ coming from the $E_{8}$ del Pezzo corresponding to $W$. In this setting the stable degeneration consists of two copies of $J$ which are identified along $E$ sitting inside each of them only the gluing map is not the identity on $E$ but rather the automorphism $t_{\alpha}: E \rightarrow E$. We will denote the resulting normal crossing surface by $J \coprod_{E, t_{\alpha}} J$. By construction $J \coprod_{E, t_{\alpha}} J$ has a fixed point free involution which interchanges the two copies of $J$ and acts as $t_{\alpha}$ on the common curve $E$.

\subsection{The quotient model}

If one thinks heuristically about the CHL string as a Heterotic string with involution one expects that the duality should produce an F-theory compactified on a K3 with involution. Both on-the-nose correspondence and its stable limit have this property but nevertheless the duality they produce is not completely satisfactory. One way to see that is to observe that if the pair $(S, e)$ is to correspond to the CHL string, then the corresponding F-theory will have to be compactified on the variety $S /\langle e\rangle$. This however immediately runs into a problem since $S /\langle e\rangle$ is an Enriques surface and hence cannot be Ricci flat. This seeming contradiction is resolved if we go back and carefully compare the action of the Enriques involution $e$ and the CHL involution $i$ on the Narain lattice Nar $\subset \Lambda$. Recall that (up to a rescaling by 2 on the 
second factor) we identified the CHL lattice $\mathrm{Nar}^{\langle i\rangle}=\mathbf{U} \oplus \mathbf{U} \oplus \mathbf{E}_{8}(2)$ with the transcendental lattice $\mathbf{U} \oplus \mathbf{U}(2) \oplus \mathbf{E}_{8}(2)$ of $S$ which in turn is identified with the lattice of anti-invariants of $e$ acting on $\Lambda$. In particular, we see that the action of $i$ on the Narain lattice matches ${ }^{11}$ the action of the involution $-e$ (rather than that of $e$ ). Therefore if we want to identify the action of the CHL involution on the F-theory side we need to find a geometric realization of the involution $-e$, that is we need to find an involution $\sigma: S \rightarrow S$ which on the Narain part of the cohomology of $S$ induces exactly ${ }^{12}$ the involution $-e$.

This can be easily done if we remember that by virtue of the Heterotic/Ftheory duality the surface $S$ comes equipped with an elliptic fibration with a section. Let inv denote the involution on $S$ which acts as inversion along the elliptic fibers. We will see that inv and $e$ commute as automorphisms of $S$ and that the composition $\sigma:=\operatorname{inv} \circ e$ acts as $-e$ on the Narain lattice $\operatorname{Nar} \subset \Lambda$.

The key is understanding the relationship of $\sigma$ and the Enriques surface $F=S /\langle e\rangle$. Since the elliptic fibration structure on $S$ induces an elliptic fibration $a: F \rightarrow \mathbf{P}^{1}$ on $F$ one can change the viewpoint and consider both $S$ and $\sigma$ as attributes of $F$ and its elliptic fibration structure. For this we will need some preliminaries on the moduli space of elliptic Enriques surfaces.

It is well known (see [4], [10], Chapter V or [16], Section 4.5) that every Enriques surface admits an elliptic pencil. Since the general Enriques surface has an infinite automorphism group (see [3]) this implies that the general Enriques surface admits infinitely many elliptic structure. However only finitely many ${ }^{13}$ of those are inequivalent modulo automorphisms and so the moduli space of elliptic Enriques surfaces is just a finite cover of the moduli of Enriques surfaces. Every elliptic fibration $a: F \rightarrow \mathrm{P}^{1}$ on an Enriques surface $F$ has exactly two multiple ${ }^{14}$ fibers (see e.g. [16], Section 4.5). In particular a cannot have a section but it is known that $a$ admits a 2-section of arithmetic genus 0 or 1 . The relative Jacobian $a^{0}: J^{0}\left(F / \mathbf{P}^{1}\right) \rightarrow \mathbf{P}^{1}$ of $a$ is an elliptic surface

\footnotetext{
${ }^{11}$ After embedding $\operatorname{Nar} \subset \Lambda$ as $\left(u, v, \xi^{\prime}, \xi^{\prime \prime}\right) \mapsto\left(u, v,-v, \xi^{\prime}, \xi^{\prime \prime}\right)$.

${ }^{12} \mathrm{Up}$ to a choice of marking.

${ }^{13} 527$ to be precise - see [3].

${ }^{14}$ Recall that a fibration $f: \Sigma \rightarrow B$ of a smooth variety $\Sigma$ over a curve $B$ is said to have an $m$-tuple fiber at a point $b \in B$ if the pullback $f^{*} u$ of a local coordinate $u$ at $b$ vanishes to order $m$ at every point of $f^{-1}(b)$.
} 
with a section and twelve $I_{1}$-fibers, i.e. a rational elliptic surface [10]. A remarkable construction of Kodaira - the so called logarithmic transform ${ }^{15}$ - allows one to reconstruct $F$ from $J^{0}\left(F / \mathbf{P}^{1}\right)$ and a little bit of extra data. In this case the extra data needed is a choice of two smooth elliptic fibers of the rational elliptic surface $J^{0}\left(F / \mathbf{P}^{1}\right)$ and a choice of a point of order two on each of them. To summarize - the moduli space of elliptic Enriques surfaces can be identified with the moduli space of triples $\left(J,\left(E_{1}, \alpha_{1}\right),\left(E_{2}, \alpha_{2}\right)\right)$ where $J$ is a rational elliptic surface, $E_{1}$ and $E_{2}$ are two anticanonical curves on $J$ with marked origin and and $\alpha_{i} \in E_{i}[2]$ are points of order two. Since every rational elliptic surface is the blow-up of $\mathbf{P}^{2}$ in the nine intersection points of two cubic curves, we obtain an equivalent description of the moduli space as the space parameterizing pairs of plane cubics with a choice of a point of order two on each of them. The rational elliptic surface $J$ corresponding to $F$ is exactly the one appearing in the stable degeneration of $S$ and pair $(E, \alpha)$ appearing in the CHL data determining $F$ is isomorphic to one of the two pairs $\left(E_{1}, \alpha_{1}\right),\left(E_{2}, \alpha_{2}\right)$. Thus the moduli of CHL data is actually isomorphic to the moduli space of elliptic Enriques surfaces with a marked double fiber.

With this in mind we can now go back and reexamine the involution $\sigma: S \rightarrow S$. The data of $F$ and its elliptic fibration $a: F \rightarrow \mathbf{P}^{1}$ determines two points on the base $\mathbf{P}^{1}$ - namely the points where the double fibers of $a$ sit. For $B \cong \mathbf{P}^{1} \rightarrow \mathbf{P}^{1}$ - the double cover branched at those two points we can form the fiber product $F \times_{\mathbf{P}^{1}} B$ which is not

${ }^{15}$ The logarithmic transform is a general procedure which produces multiple fibers in elliptic fibrations. Here is a brief description of how the logarithmic transform works (see [16] for more details). Start with an elliptic surface $f: \Sigma \rightarrow B$, a point $b \in B$ and a non-trivial $m$-torsion point $\alpha_{b} \in \Sigma_{b}$. The existence of $\alpha_{b}$ implies that $\Sigma_{b}$ has a non-trivial fundamental group, i.e. that $\Sigma_{b}$ is of type $I_{k}, k \geq 0$. For simplicity we assume that $k=0$, i.e. that $\Sigma_{b}$ is a smooth elliptic curve. If $b \in \Delta \subset B$ is a small analytic disk, then $\Sigma_{\mid \Delta} \rightarrow \Delta$ is a topologically trivial elliptic fibration since $\Delta$ is simply connected. In particular the cover of $m$-torsion points splits over $\Delta$ and so there is a unique analytic section $\alpha: \Delta \rightarrow \Sigma_{\mid \Delta}$ of $m$-torsion points passing trough $\alpha_{b}$. Let $p_{m}: \Delta_{m} \rightarrow \Delta$ be the degree $m$ cyclic cover of $\Delta$ branched at $b$. The multiplication by an $m$-th root of unity on $\Delta_{m}$ lifts to an automorphism $\phi: p_{m}^{*}\left(\Sigma_{\mid \Delta}\right) \rightarrow p_{m}^{*}\left(\Sigma_{\mid \Delta}\right)$ (of order $m$ ). The translation $t$ by the (pull-back) of the section $\alpha$ is also an automorphism of order $m$ of $p_{m}^{*}\left(\Sigma_{\mid \Delta}\right)$ and the quotient $\left(p_{m}^{*}\left(\Sigma_{\mid \Delta}\right)\right) /\langle\phi \circ t\rangle$ is an analytic surface isomorphic to $\Sigma_{\mid \Delta}$ over the punctured disk $\Delta-\{b\}$ and having an $m$-tuple fiber at $b$. By gluing back this quotient to $\Sigma-\Sigma_{b}$ one obtains a new complex surface $\log \left(\Sigma, b, \alpha_{b}\right)$ which is analytically isomorphic to $\Sigma$ over $B-\{b\}$ and has a multiple fiber at $b$. 
normal along the curves over the two marked points. The normalization $\left(F \times_{\mathbf{p}^{1}} B\right)^{\nu}$ is a smooth non-ramified double cover of $F$ which is easily seen to be irreducible and so coincides with $S$. If $x \in \mathbf{P}^{1}$ is a point different from the two marked points the preimage of $x$ in $B$ consists of two points $b^{\prime}$ and $b^{\prime \prime}$ and the preimage of $x$ in $S$ consists of two copies $E^{\prime}$ and $E^{\prime \prime}$ of the fiber $F_{x}$. The Enriques involution $e$ acts by interchanging $E^{\prime}$ and $E^{\prime \prime}$ and the inversion acts on $E^{\prime}$ and $E^{\prime \prime}$ separately. In particular $e$ and inv commute outside of the preimages of the double fibers and since these are global automorphisms they must commute everywhere. This implies that $\sigma=e \circ \mathbf{i n v}=\mathbf{i n v} \circ e$ is an involution on $S$ and by the description just given we see that $\sigma$ acts without fixed points outside of the preimages of the two double fibers in $F$. Furthermore the action of $\sigma$ leaves each of the two preimages of the double fibers and has for fixed points on each of them. To understand what the quotient $X:=S /\langle\sigma\rangle$ looks like note that since both $e$ and inv act as multiplication by -1 on the holomorphic symplectic form $\Omega$ on $S$, the composition $\sigma$ will leave $\Omega$ invariant and so $X$ will be a K3 surface with 8 singularities of type $A_{1}$. Moreover since the elliptic fibration on $S$ has a section one knows that the quotient $S /\langle\mathbf{i n v}\rangle$ is isomorphic to the Hirzebruch surface $\mathbf{F}_{4}$. This implies that the sublattice of invariants $H^{2}(S, \mathbf{Z})^{\langle\mathbf{i n v}\rangle}$ can be identified with the pull-back of the second cohomology of $\mathbf{F}_{4}$ and is hence coincides with the sublattice $\mathbf{U} \subset H^{2}(S, \mathbf{Z})$ spanned by the elliptic fiber and the section. In particular inv acts as multiplication by -1 on the transcendental lattice of $S$ and therefore $\sigma=e$ o inv acts as $-e$ on the Narain part of the cohomology of $S$.

As explained in the beginning of the subsection this is exactly the condition that an involution on $S$ should satisfy in order to match the CHL involution. Thus the F-theory corresponding to the CHL string is naturally compactified on the $\mathrm{K} 3$ surface $X=S /\langle\sigma\rangle$. The CHL lattice $\mathbf{N a r}^{\langle i\rangle}=\mathbf{U} \oplus \mathbf{U} \oplus \mathbf{E}_{8}$ appears in this context as the intersection of the invariants of the $\sigma$ action on $H^{2}(S, \mathbf{Z})$ and the transcendental lattice of $S$. In other words the CHL latice becomes exactly the (pullback to $S$ ) of the transcendental lattice of $X$. Alternatively we may blow up the eight $A_{1}$ singularities of $X$ to obtain a smooth K3 surface $\hat{X}$ whose Picard lattice contains the even non-degenerate lattice $N$ of signature $(1,9)$. Explicitly the elliptic fibration on $S$ descends to an elliptic fibration on $p: X \rightarrow \mathbf{P}^{1}$ with two special fibers which are double $\mathbf{P}^{1}$ 's each carrying four of the eight singular points. The elliptic fibration $p$ does not have a section but has a double section and so the 
elliptically fibered K3 surface $\hat{p}: \hat{X} \rightarrow \mathbf{P}^{1}$ has two fibers of type $I_{0}^{*}$ $\left(=\hat{D}_{4}\right)$ and a double section intersecting the double component of each $I_{0}^{*}$ fiber at one point. Therefore the lattice $N \subset \operatorname{Pic}(\hat{X})$ is generated by the ten components of the two $I_{0}^{*}$ fibers and the double section of $\hat{p}$. In this way the moduli space of F-theory compactifications dual to the CHL string becomes naturally isomorphic to the moduli space of all $\mathrm{N}$-polarized K3 surfaces or equivalently to the moduli space of all elliptic K3 surfaces with two $I_{0}^{*}$ fibers and a double section which recovers Witten's description from [27].

To justify the last identification we have to exhibit a way of reconstructing $S$ from the surface $X$. To do that consider the resolved model $\hat{p}: \hat{X} \rightarrow \mathbf{P}^{1}$ and let $p_{1}, p_{2} \in \mathbf{P}^{1}$ be the two points over which we have the two $I_{0}^{*}$ fibers. The fibers over the $p_{i}$ 's can be written as

$$
\hat{X}_{p_{i}}=2 f_{i}+e_{i}^{1}+e_{i}^{2}+e_{i}^{3}+e_{i}^{4}, i=1,2
$$

where $f_{1}, f_{2}$ and $e_{i}^{j}, i=1,2, j=1,2,3,4$ are smooth -2 curves and the $e_{i}^{j}$ s contract to the eight singular points of $X$. Consider the line bundle $L=\mathcal{O}_{\hat{X}}\left(\sum_{j=1}^{4}\left(e_{1}^{j}+e_{2}^{j}\right)\right)$ on $\hat{X}$. By 9 we have $L=\mathcal{O}_{\hat{X}}\left(\hat{X}_{p_{1}}+\right.$ $\left.\hat{X}_{p_{2}}-2 f_{1}-2 f_{2}\right)=\hat{p}^{*} \mathcal{O}_{\mathbf{P}^{1}}(2) \otimes \mathcal{O}_{\hat{X}}\left(-2 f_{1}-2 f_{2}\right)$ and so $L$ has a unique square root equal to $\hat{p}^{*} \mathcal{O}_{\mathbf{P}^{1}}(1) \otimes \mathcal{O}_{\hat{X}}\left(-f_{1}-f_{2}\right)$. Due to this there exists a unique double cover $\hat{S} \rightarrow \hat{X}$ which is simply branched exactly at the eight curves $e_{i}^{j}$. The ramification divisor of the cover $\hat{S} \rightarrow \hat{X}$ consists of eight smooth rational curves $\hat{e}_{i}^{j}$ mapping isomorphically to the branch curves $e_{i}^{j}$. Since the $\hat{e}_{i}^{j}$, s are part of the ramification divisor we have $\left(2 \hat{e}_{i}^{j}\right)^{2}=2\left(e_{i}^{j}\right)^{2}=-4$ and hence $\left(\hat{e}_{i}^{j}\right)^{2}=-1$ for all $i, j$. Therefore we can contract all of the $\hat{e}_{i}^{j}$ 's to obtain a smooth K3 surface $S$.

Remark. The Enriques surface $F$ played an auxiliary role in the duality construction above and will not appear in our considerations from now on. It is worth mentioning however that by replacing the Enriques involution $e$ by the involution $\sigma$ one introduces a finite ambiguity. More precisely, the moduli space of pairs $(S, e)$ is a finite cover of the moduli space of pairs $(S, \sigma)$. Indeed - to reconstruct a pair $(S, e)$ from a pair $(S, \sigma)$ one needs also to specify points of order two along the two $\sigma$-invariant fibers of the Weierstrass elliptic fibration on $S$. 


\subsection{The $\Gamma_{0}(2)$-model}

As we have just seen the F-theory dual to the CHL string lives naturally on an elliptic K3 surface $X$ of a special kind. The description we have obtained however is still not completely satisfactory since as we saw the elliptic fibration structure $p: X \rightarrow \mathbf{P}^{1}$ that $X$ inherits from the duality with the CHL string does not admit a section but only a double section. This causes a problem since in view of the duality with the IIB string the F-theory moduli should all be encoded into data living entirely on the base of the elliptic fibration, i.e. the F-theory should be compactified on a Weierstrass elliptic fibration. One can try to remedy the lack of section for $p: X \rightarrow \mathbf{P}^{1}$ by replacing $X$ by its (basic) relative Jacobian fibration ${ }^{16} J^{0}\left(X / \mathbf{P}^{1}\right) \rightarrow \mathbf{P}^{1}$ which always has a Weierstrass form and so is encoded into geometric data on $\mathbf{P}^{1}$ only. This is not a big sacrifice to make since $X$ is always algebraic and so according to Kodaira's (see [18]) and Ogg-Shafarevich's (see [10] , Section V.3) theory the surface $X$ is determined by the surface $J^{0}\left(X / \mathbf{P}^{1}\right)$ up to finite data. On the other hand if the deformation theory of the surface $X$ is governed by a Weierstrass eqution only we should be seen an 18 dimensional moduli space rather than a 10 dimensional one since there is no obstruction to deforming a K3 surface in Weierstrass form away from the locus of elliptic fibrations having two $I_{0}^{*}$ fibers. Therefore it is necessary to find a mechanism that is intrinsic to F-theory that will allow the confinement of the moduli of the elliptic fibration to the subfamily we get trough the duality with the CHL string.

One instance in which this will necessarily happen is if there is an additional field in F-theory whose deformations are obstructed in the full moduli of elliptic K3 surfaces. The only field which is intrinsically present in the F-theory and which has been set to equal zero in order for the full F-theory/Heterotic duality to work is the skew-symmetric B-field on the base of the F-theory compactification. So it is reasonable to expect that in a consistent realization of the $\mathrm{F}$-theory vacua on $X$ one will have to describe not only the dilaton-axion fields but also a B-field which constrains the deformations of the compactification. To that end we examine how the presence of a non-trivial B-field is encoded in the geometry of an F-theory compactification.

\footnotetext{
${ }^{16}$ Which turns out to be a K3 surface as well.
} 
Let $a: C Y \rightarrow S$ be an elliptically fibered Calabi-Yau manifold on which F-theory is compactified. Fix a point $o \in S^{o}:=S \backslash$ Discriminant in the complement of the discriminant of $a$. Let $E=a^{-1}(o)$ be the fiber of $a$ over $o$ and let mon $: \pi_{1}\left(S^{o}, o\right) \rightarrow S L(2, \mathbf{Z}) \subset G L\left(H^{1}(E, \mathbf{Z})\right)$ be the monodromy representation of the family $X \rightarrow S^{o}$. The data describing a B-field in the F-theory consists of a complex multivalued 2 -form $\zeta$ on $S^{o}$ which satisfies: (i) $\zeta$ becomes single valued modulo the translation action of the lattice $H^{1}(E, \mathbf{Z})$; (ii) the pair $(\zeta, \bar{\zeta})$ transforms according to the monodromy representation mon. In coordinatefree terms $\zeta$ should be viewed as a multivalued 2-form on $S^{o}$ with coefficients in the complex vector space $H^{0,1}(E)=H^{1}\left(E, \mathcal{O}_{E}\right)$ for which: (i) $\zeta$ is single valued modulo the translation action of the lattice $H^{1}(E, \mathbf{Z}) \subset H^{1}\left(E, \mathcal{O}_{E}\right)$; (ii) $(\zeta, \bar{\zeta}) \in H^{0,1}(E) \oplus H^{1,0}(E)=H^{1}(E, \mathbf{C})$ is invariant under the action of $\operatorname{mon}\left(\pi_{1}\left(S^{o}, o\right)\right)$. Since only the cohomology class of these 2 -forms on $S$ is visible by the physics, after fixing a basis in $H^{2}(S, \mathrm{C})$ we can view the components of $\zeta$ as real multisections in $R^{1} a_{*} \mathbf{C} \otimes H^{2}(S, \mathbf{C})$ which are horizontal (w.r.t. the Gauss-Manin connection) and become single valued modulo the action of $R^{1} a_{*} \mathbf{Z} \subset R^{1} a_{*} \mathbf{C}$. This implies in particular that when projected to $R^{1} a_{*} \mathcal{O}_{X} / R^{1} a_{*} \mathbf{Z} \subset C Y$ each component of $\zeta$ becomes a holomorphic (single valued) section of the elliptic fibration $a: C Y \rightarrow S$ which is horizontal ${ }^{17}$ for the Gauss-Manin connection on the family of elliptic curves $C Y_{\mid S^{\circ}} \rightarrow S^{o}$. Conversely, any such section of $a$ will determine the component of a B-field.

The situation simplifies somewhat if we are in the case where $h^{2}(S, \mathbf{C})=$ 1. In this case the B-field $\zeta$ itself is realized as a holomorphic section of $a: C Y \rightarrow S$ which is Gauss-Manin horizontal. If the Calabi-Yau manifold $C Y$ is general in the sense that $\operatorname{mon}\left(\pi_{1}(S, o)\right)=S L(2, \mathrm{Z})$, then the only such section will be the zero section of the elliptic fibration. So generically the B-field will be zero. Moreover it is not hard to see that the only horizontal sections for the Gauss-Manin connection will have to be sections representing torsion points on the elliptic fiber. Thus the only situations when one can turn-on the B-field in the F-theory compactified on $C Y$ is when the monodromy group $\operatorname{mon}\left(\pi_{1}(S, o)\right)$ preserves some vector in $\frac{1}{n} H^{1}(E, \mathbf{Z}) \subset H^{1}(E, \mathbf{Q})$, i.e. when $\operatorname{mon}\left(\pi_{1}(S, o)\right)$ is contained in some congruence subgroup of level $n$.

Going back to the compactification on the special K3 surface $X$ one

${ }^{17} \mathrm{Or}$ in other words is a locally constant normal function in the sense of [15]. 
can observe that the inherited elliptic fibration $p: X \rightarrow \mathbf{P}^{1}$ will have general monodromy and so one cannot turn on the B-field neither for the fibration on $X$ nor for the fibration on $J^{0}\left(X / \mathrm{P}^{1}\right)$. Therefore, in order to obtain a consistent description for the F-theory vacua on $X$ we are forced to look for a different elliptic structure on $X$ which has monodromy contained in a congruence subgroup. We find a natural elliptic structure $\pi: X \rightarrow \mathbf{P}^{1}$ like that which turns out to have monodromy contained in the congruence subgroup $\Gamma_{0}(2)$. This means that $\pi$ admits a section consisting of 2-torsion points or equivalently that the F-theory compactified on $X$ with the elliptic fibration $\pi$ admits a non-trivial B-field corresponding to $1 / 2$ the Fubini-Studi form on $\mathbf{P}^{1}$. As a consistency check we study the moduli space of deformations of this elliptic fibration that preserve the B-field and show that it is isogeneous to the moduli space of $X$ considered as a $N$-polarized K3 surface. Further evidence for the relevance of the $\Gamma_{0}(2)$ fibration was provided in Section 3 where we describe an effective mechanism allowing one to read the spectrum of gauge groups for the CHL string in terms of the elliptic fibration $\pi$.

The construction of the fibration $\pi: X \rightarrow \mathbf{P}^{1}$ or equivalently its lift $\hat{\pi}: \hat{X} \rightarrow \mathbf{P}^{1}$ to the resolution of $X$ is based on a specific projective model of $X$ which we explore next.

We have already seen one explicit model of $S$ and $X$ in Section 2. There $S$ was given in its Weierstrass form 4 and $X$ was described as the quotient of $S$ by the involution $\sigma(x, y: z)=(x,-y ; 1 / z)$. The elliptic fibration on $S$ descends through this construction to the inherited elliptic fibration $p: X \rightarrow \mathbf{P}^{1}$. To obtain the $\Gamma_{0}(2)$ elliptic fibration $\pi: X \rightarrow \mathbf{P}^{1}$ we will construct $X$ in a different way - namely as a double cover of the Hirzebruch surface $Q:=\mathbf{F}_{0}=\mathbf{P}^{1} \times \mathbf{P}^{1}$. It is well known that all Enriques surfaces can be realized as double covers of $Q$ [10]Tंhis suggests that the surfaces $X$ can also be realized as double covers of quadrics which is indeed the case.

In order for a double cover of $Q$ to be a $\mathrm{K} 3$ surface it has to be branched along a curve of type $(4,4)$, i.e. along the divisor of a section in the line bundle $\mathcal{O}_{Q}(4,4)$. Every such cover will have two natural elliptic fibrations corresponding to the two rulings of the quadric $Q$, that is to the projections $q_{1}: Q \rightarrow \mathbf{P}^{1}$ and $q_{2}: Q \rightarrow \mathbf{P}^{1}$ to the two factors of $Q$. If we want to have two $I_{0}^{*}$ fibers in say the fibration 
corresponding to $q_{1}$ we are necessarily forced to consider only double covers of $Q$ branched along curves of the form $r_{1} \cup r_{2} \cup C$ where the $r_{i}$ 's are two fibers of $q_{1}$ and $C$ belongs to the linear system $\mathcal{O}_{Q}(2,4)$. The number of moduli of such K3's is easily seen to be 10 since we have $\operatorname{dim} \mathbf{P}\left(H^{0}\left(Q, q_{1}^{*} \mathcal{O}_{\mathbf{P}^{1}}(2)\right)\right)=\operatorname{dim} \mathbf{P}\left(H^{0}\left(\mathbf{P}^{1}, \mathcal{O}_{\mathbf{P}^{1}}(2)\right)\right)=2$ parameters in the $r_{i}$ 's and $\operatorname{dim} \mathbf{P}\left(H^{0}\left(Q, \mathcal{O}_{Q}(2,4)\right)\right)=\operatorname{dim} \mathbf{P}\left(H^{0}\left(\mathbf{P}^{1}, \mathcal{O}_{\mathbf{P}^{1}}(2)\right) \otimes\right.$ $\left.H^{0}\left(\mathbf{P}^{1}, \mathcal{O}_{\mathbf{P}^{1}}(4)\right)\right)=3 \cdot 5-1=14$ parameters in the curves $C$ and we also have the 6 dimensional group of automorphisms of $Q$. Due to this the moduli space of $N$-polarized K3 surfaces $X$ is isomorphic to the moduli space of double covers of $Q$ branched along curves of the form $r_{1}+r_{2}+C$ and so each $X$ has a second elliptic fibration $\pi: X \rightarrow \mathbf{P}^{1}$ corresponding to the projection $q_{2}: Q \rightarrow \mathbf{P}^{1}$.

The elliptic fibration $\pi$ has two sections $f_{1}$ and $f_{2}$, each corresponding to the double component of the two $I_{0}^{*}$ fibers in the elliptic fibration $p$. Each of the $f_{i}$ 's passes trough four of the $A_{1}$ singularities of $X$ as seen on Figure 4. On the resolved surface $\hat{X}$ then one sees that the elliptic fibration $\hat{\pi}: \hat{X} \rightarrow \mathbf{P}^{1}$ has eight fibers of type $I_{2}$ and two sections arranged in such a way that at each $I_{2}$ fiber the two sections pass trough two different components of the fiber. It is not hard now to obtain a representation of the moduli space of $X$ 's as a Weirestrass family - namely we can declare the section $\hat{f}_{1}$ to be the zero section and contract the components in the $I_{2}$ fibers not meeting it. The resulting surface $Y$ will be an elliptic K3 surface with two sections and eight $A_{1}$ singularities sitting on one of them. Moreover if we examine what this operation does to the realization of $X$ as a double cover of the quadric $Q$ we see that the passage from $X$ to $Y$ just amounts to performing 4 elementary modifications of $Q$ at the four points of intersection of $r_{1}$ and $C$. In other words on $Q$ we will have to blow up the four points $\left\{x_{1}, x_{2}, x_{3}, x_{4}\right\}=C \cap r_{1}$ and then contract the $q_{2}$-fibers $q_{2}^{-1}\left(q_{2}\left(x_{1}\right)\right), q_{2}^{-1}\left(q_{2}\left(x_{2}\right)\right), q_{2}^{-1}\left(q_{2}\left(x_{3}\right)\right), q_{2}^{-1}\left(q_{2}\left(x_{4}\right)\right)$ passing trough these points. Since each elementary modification like that increases the index of the Hirzebruch surface by one at the end of the day we will get a realization of $Y$ as a double cover of the Hirzebruch surface $\mathbf{F}_{4}$. In particular the $\mathrm{K} 3$ surface $Y$ is in a Weierstrass form so that the section $f_{1}$ sits over the section at infinity ${ }^{18}$ in $\mathbf{F}_{4}$ and is thus interpretted as the zero section of the elliptic fibration $w: Y \rightarrow \mathbf{P}^{1}$. The curve of points of order two for $w$ is then naturally identified with

\footnotetext{
${ }^{18}$ i.e. the unique section of self intersection -4 .
} 
the ramification divisor of the double cover $Y \rightarrow \mathbf{F}_{1}$ and consequently coincides with the (proper transform) of the curve $f_{1} \cup f_{2} \cup C$. Due to this we conclude that both $w: Y \rightarrow \mathbf{P}^{1}$ and $\pi: X \rightarrow \mathbf{P}^{1}$ have monodromy contained in $\Gamma_{0}(2)$ and so the second section $f_{2}$ of $w$ should be viewed as the B-field for the F-theory compactification ${ }^{19}$.

Finally we describe the deformations of the Weierstrass fibration $w: Y \rightarrow \mathbf{P}^{1}$ in which the second section $f_{2}$ survives. For that one just needs to observe that all such deformations are coming as double covers of $\mathbf{F}_{4}$ branched along a curve of the form $e_{\infty}+e_{0}+C$ where $e_{\infty}$ is the infinity section of $\mathbf{F}_{4}, e_{0}$ is a section in $\mathcal{O}_{\mathbf{P}^{1}}(4) \subset \mathbf{P}\left(\mathcal{O}_{\mathbf{P}^{1}} \oplus \mathcal{O}_{\mathbf{P}^{1}}(4)\right)$ and $C$ is a curve in the linear system $\left|2 e_{\infty}+8 f\right|$ ( $f$ is the fiber of $\mathbf{F}_{4} \rightarrow \mathbf{P}^{1}$. The section $e_{0}$ is then given by a degree four polynomial $a_{4}(z)$ on $\mathbf{P}^{1}$ and so the Weierstrass equation of $Y$ must be of the form 6 we used in Section 3.

\subsection{Matching of collision patterns}

The representation of the surface $X$ as double covers of a quadric is very useful not only for finding the $\Gamma_{0}(2)$ elliptic fibration on $X$ but also for analyzing the gauge groups appearing after several singular fibers of $\pi$ collide in the presence of a non-trivial B-field. Here we show how to carry such an analysis in several model cases. This information was used extensively in Section 3 where we described the gauge group spectrum of the F-theory with fluxes on $X$.

The basic idea which makes the matching of singularities possible is the observation that the singular fibers of either the inherited or the $\Gamma_{0}(2)$ elliptic fibrations on $X$ will correspond to certain singularities of the branch curve $r_{1} \cup r_{2} \cup C$. Thus we may describe the Kodaira fibers (in either fibration) on the minimal resolution of $X$ by just resolving the singularities on the branch curve and keeping track of what part of the exceptional locus will have to enter the resolved branch divisor.

$S U(n)$ gauge groups: As usual we are interested only in non-abelian gauge groups so we are in the case when $n \geq 2$. According to our recipe the $S U(n)$ gauge groups appear when $n+1$ fibers of type $I_{1}$ in

\footnotetext{
${ }^{19}$ Note that both $X$ and $Y$ have a common resolution $\hat{X}$ on $X$.
} 
the inherited elliptic fibration collide with each other and the collision occurs away from the two $I_{0}^{*}$ fibers. In terms of the branch curve this just means that the curve $C$ acquires a higher order of contact with a ruling $f$ of $p_{2}: Q \rightarrow \mathbf{P}^{1}$ which is different from $r_{1}$ and $r_{2}$. Since we want our gauge groups to be localized at a single fiber in both fibration we will further assume that the high order of contact of $C$ and $f$ occurs at a single point of $f$.

For example a $I_{1}$ fiber in the inherited elliptic fibration corresponds to $C$ being tangent to $f$ at one point $x$ and (since $f \cdot C=4$ ) necessarily intersecting it at two more points $x_{1}, x_{2}$. The corresponding $I_{1}$ fiber of $\pi: X \rightarrow \mathbf{P}^{1}$ is then just the double cover of $f$ branched at the divisor $C \cdot f=2 x+x_{1}+x_{2}$.

To get the group $S U(2)$ we need to look at the case when $C \cdot f$ contains a point $x$ with multiplicity 2 so that $C$ has a node at $x$. Figure 5 shows how the minimal resolution of $X$ is obtained by resolving the singularities of the branch divisor and how that leads to a $I_{2}$ Kodaira fiber in the inherited elliptic fibration. The branch and ramification divisors are represented by the thick solid lines and $f$ and $\phi$ the fibers of the inherited and $\Gamma_{0}(2)$ fibrations respectively as well as their projection to $Q$. The same singularity viewed from the point of view of the $\Gamma_{0}(2)$ fibration is shown on Figure 6.

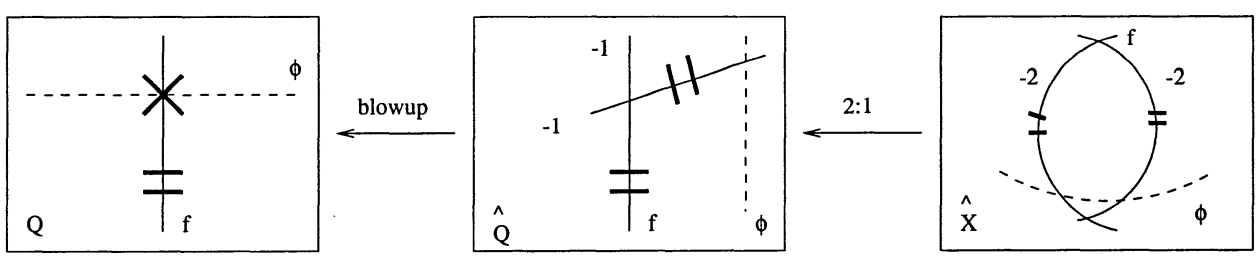

Figure 5: $S U(2)$ in the inherited elliptic fibration.

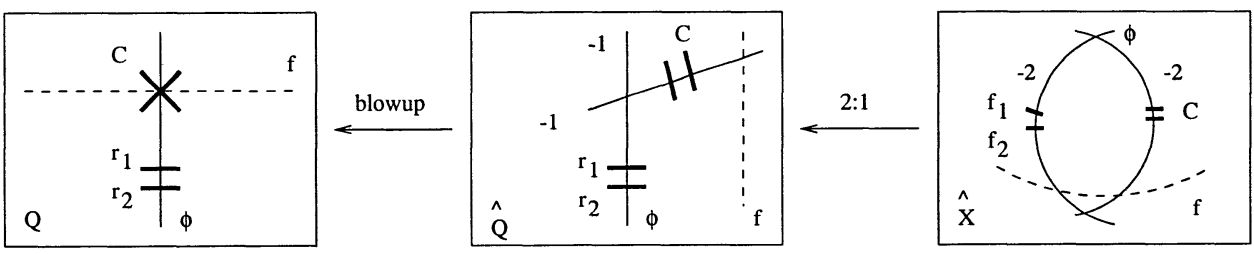

Figure 6: $S U(2)$ in the $\Gamma_{0}(2)$ elliptic fibration.

In exactly the same way a $S U(3)$ gauge group will appear when $C$ 
acquires a cusp at some point $x \in f$.

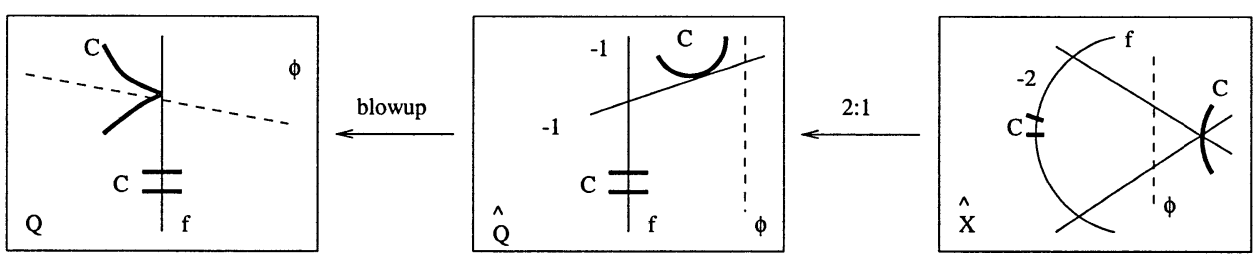

Figure 7: $S U(3)$ in the inherited elliptic fibration.

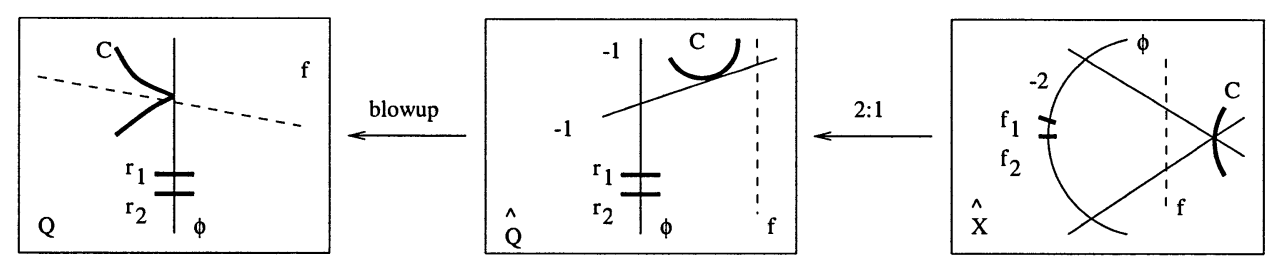

Figure 8: $S U(3)$ in the $\Gamma_{0}(2)$ elliptic fibration.

The groups $S U(n)$ for $n>3$ are obtained in exactly the same way after resolution of the appropriate singularities of $C$.

$S p(n)$ gauge groups: As explained in Section 2 the non-simply laced gauge groups appear as quotients of Dynkin diagrams of curves on $S$ by the involution $\sigma$ acting as an exterior automorphisms. In the inherited elliptic fibration on $S$ this situation corresponds to collections of $2 n I_{1}$ fibers symmetric with respect to $\sigma$ colliding at one of the $\sigma$-invariant fibers. In the inherited elliptic fibration on $X$ this corresponds to $n I_{1}$ fibers colliding on top of one of the $I_{0}^{*}$ fibers and so on $Q$ this is seen as a higher order of contact of the curve $C$ with one of the rulings entering the branch divisor. For concreteness we will assume that this ruling is $r_{1}$.

The first non-abelian case of such collision will occur when the curve $C$ becomes tangent to $r_{1}$ at one point. The necessary blowups and the resulting Kodaira fibers in both elliptic fibrations can be seen on Figures 9, 10. 


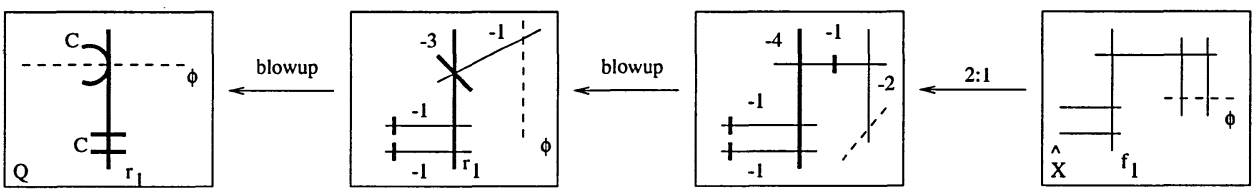

Figure 9: Collision leading to $S p(1)$ (inherited fibration).

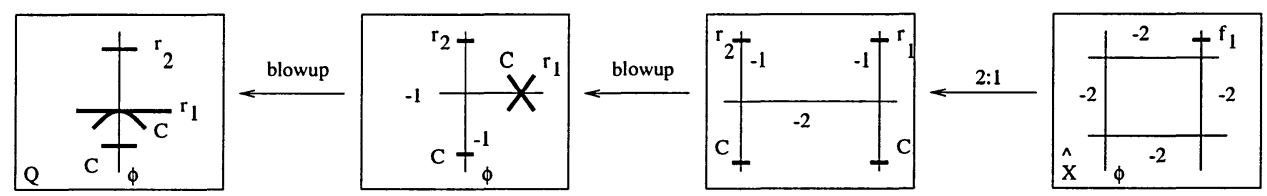

Figure 10: Collision leading to $S p(1)\left(\Gamma_{0}(2)\right.$ fibration).

As one can see from these blowups the Kodaira fibers corresponding to this simplest collision are of type $\hat{D}_{5}$ in the inherited elliptic fibration and of type $\hat{A}_{3}$ in the $\Gamma_{0}(2)$ elliptic fibration. To see the actual gauge group we have to trace the behavior of the -2 curves comprising these Kodaira fibers on the double cover $S \rightarrow X$. A convenient way to record what is happening is to combine both Kodaira fibers described above in one graph. This graph is shown on Figure 11 where the nodes corresponding to the inherited fiber are marked as squares and the nodes corresponding to the $\Gamma_{0}(2)$ fiber are marked as circles. The four nodes that are shadowed out are the exceptional curves corresponding to the four fixed points of $\sigma$ on the preimage of $r_{1}$ in $S$.

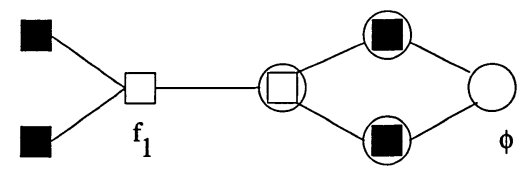

Figure 11: Combined Kodaira fibers producing $S p(1)$.

In particular we see that of the four cycles of the $\hat{A}_{3}\left(=I_{4}\right)$ fiber in the $\Gamma_{0}(2)$-fibration two are coming from fixed points for $\sigma$ and hence do not contribute to the gauge symmetry, one (marked $\phi$ ) intersects the zero section and also does not contribute and so there is only a rank one contribution to the gauge group from the one remaining cycle. Since the remaining cycle intersects with two of the cycles corresponding to fix points we see that when pulled back to $S$ it becomes an irreducible 
rational curve invariant under $\sigma$ and thus corresponds indeed to the group $S p(1)$.

The collision that produces the fibers on Figure 11 looks like colliding $2 I_{2}$ on the section $f_{1}$ from the viewpoint of the $\Gamma_{0}(2)$-fibration. Indeed - recall that the $I_{1}$ fibers of the $\Gamma_{0}(2)$-fibration appear when $C$ becomes tangent to the fibers of $q_{1}: Q \rightarrow \mathbf{P}^{1}$ and the $I_{2}$ fibers appear when $C$ intersects $R_{1}$ or $r_{2}$. In particular the collision of $2 I_{2}$ on the section $f_{1}$ will occur when $C$ becomes tangent to $r_{1}$ which is exactly the situation on Figure 10.

To see how this fits in a series we work out the next case of colliding $2 I_{2}+I_{1}$ in the $\Gamma_{0}(2)$-fibration. The necessary blowups and the corresponding Kodaira fibers for this collision are shown on Figures 12, 13.

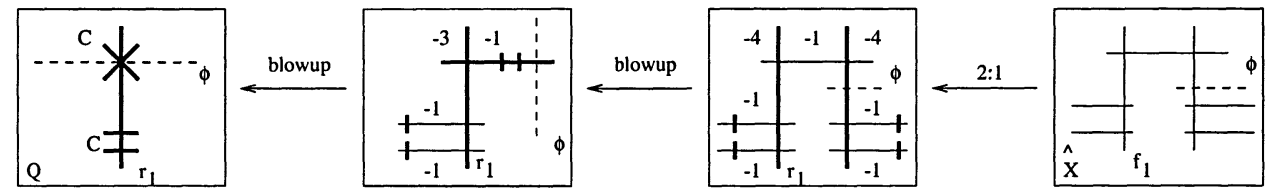

Figure 12: Collision leading to $S p(2)$ (inherited fibration).

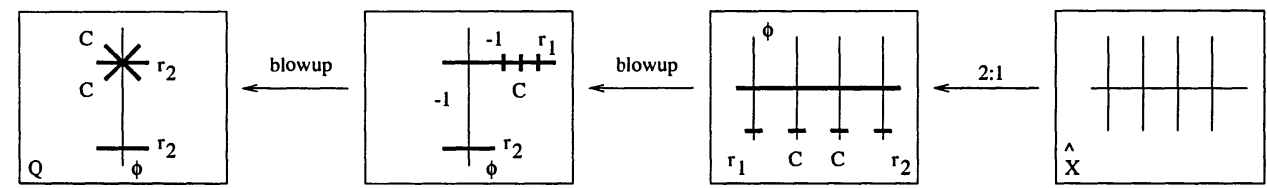

Figure 13: Collision leading to $S p(2)\left(\Gamma_{0}(2)\right.$ fibration).

As before the thick black lines denote the curves that are part of the branch divisor and so we need to blow up enough times and separate those curves to guarantee that the double cover $\hat{X}$ will be non-singular. The only new feature appearing here is that the branch divisor has a singular point with odd multiplicity (a triple point in this case) and so on the blown-up $Q$ one needs to take not only the proper transform of $C$ and the $r_{i}$ 's as branching but also one copy of the exceptional divisor in order to make the corresponding line bundle divisible by 2 .

The resulting combined Dynkin diagram is:

and we see that there are only two nodes of the $\Gamma_{0}(2)$ fiber that contribute to the gauge group and that one of them corresponds to an 


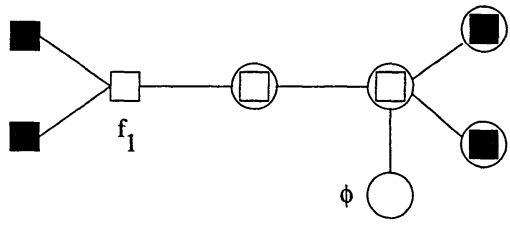

Figure 14: Combined Kodaira fibers producing $S p(2)$.

invariant sphere on $S$. Thus the $\Gamma_{0}(2)$ collision $2 I_{2}+2 I_{1}$ with the two $I_{2}$ 's coming from $\sigma$ fixed points on $f_{1}$ corresponds to a gauge group $S p(2)$.

In the same way one can work out the collisions $2 I_{2}+n I_{1}$ in the $\Gamma_{0}(2)$-fibration. The resulting combined Dynkin diagram is

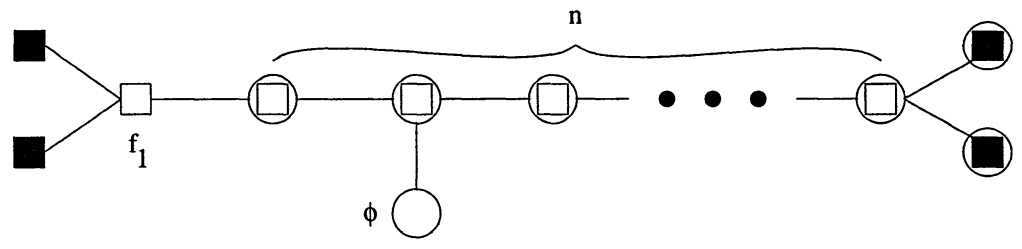

Figure 15: Combined Kodaira fibers producing $S p(n)$.

In summary - for any given collision the gauge group corresponds exactly to the Dynkin diagram consisting of the cycles which are common to the two Kodaira fibers and which do not come from fixed points of $\sigma$. Moreover the cycles intersecting pairs of exceptional divisors coming from $\sigma$-fixed points correspond to non-simply laced nodes in the diagram.

$S O(2 n)$ gauge groups: Again due to the interpretation of the CHL string as a Heterotic string with symmetry the level two $S O(2 n)$ gauge groups will appear when on $S$ we have a collection of cycles intersecting as two copies of a $D_{n}$ Dynkin diagram which are away from the invariant fibers and are swapped by $\sigma$. In terms of the inherited elliptic fibration on $X$ this just means that we have a collision of Kodaira fibers leading to a $\hat{D}_{n}$-fiber which is away from the two $I_{0}^{*}$ fibers. From the view point of the quadric $Q$ and the branch curve $C \cup r_{1} \cup r_{2}$ this collision occurs 
only when a curve of bidegree $(0,1)$ splits as a component of the curve $C$ (which has bidegree $(2,4)$ ). In other words one of the four points in $C \cap r_{1}$ becomes collinear with one of the four points in $C \cap r_{2}$. In terms of the $\Gamma_{0}(2)$ fibration this condition is expressed as a collision of two $I_{2}$ fibers coming from the two sections $f_{1}$ and $f_{2}$. Again the gauge groups can be seen as common parts of Kodaira fibers in the two elliptic fibrations but since the collision takes place away from the $\sigma$-fixed fibers, there are no shadowed nodes in the diagram and hence all the nodes are simply laced.

The simplest example is of a collision of $2 I_{2}$ fibers in the $\Gamma_{0}(2)$ fibration which come from different sections. The relevant blowup pictures are shown on Figures 16, 17.

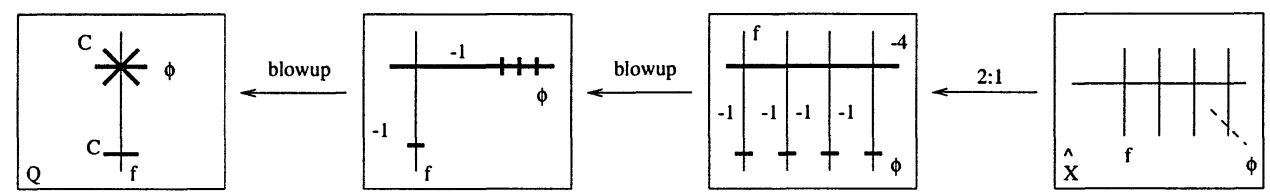

Figure 16: Collision leading to $S O(8)$ (inherited fibration).

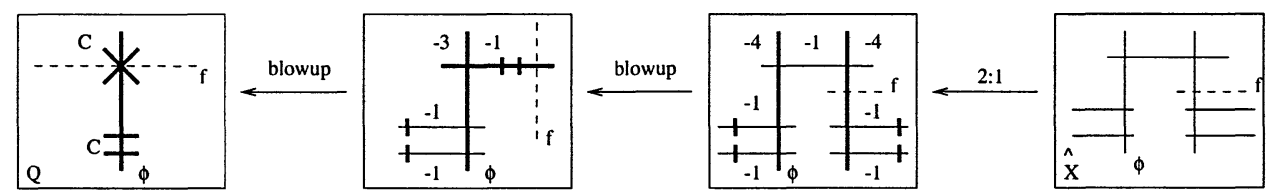

Figure 17: Collision leading to $S O(8)\left(\Gamma_{0}(2)\right.$ fibration).

As in the $S U(n)$ case, here $f$ and $\phi$ denote the fibers of the inherited and the $\Gamma_{0}(2)$ fibrations respectively (or their projections to $Q$ ). From the blowup picture we see that the Dynkin diagram of the combined Kodaira fibers is: 


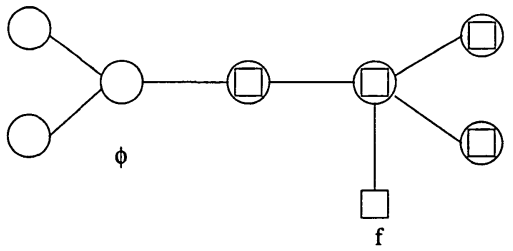

Figure 18: Combined Kodaira fibers producing $S O(8)$.

To illustrate the pattern we will work out the next case of a $2 I_{2}+2 I_{1}$ collision in the $\Gamma_{0}(2)$-fibration with the two $I_{2}$ coming from different sections. It turns out that this produces a gauge group $S O(10)$. The blow-up pictures are
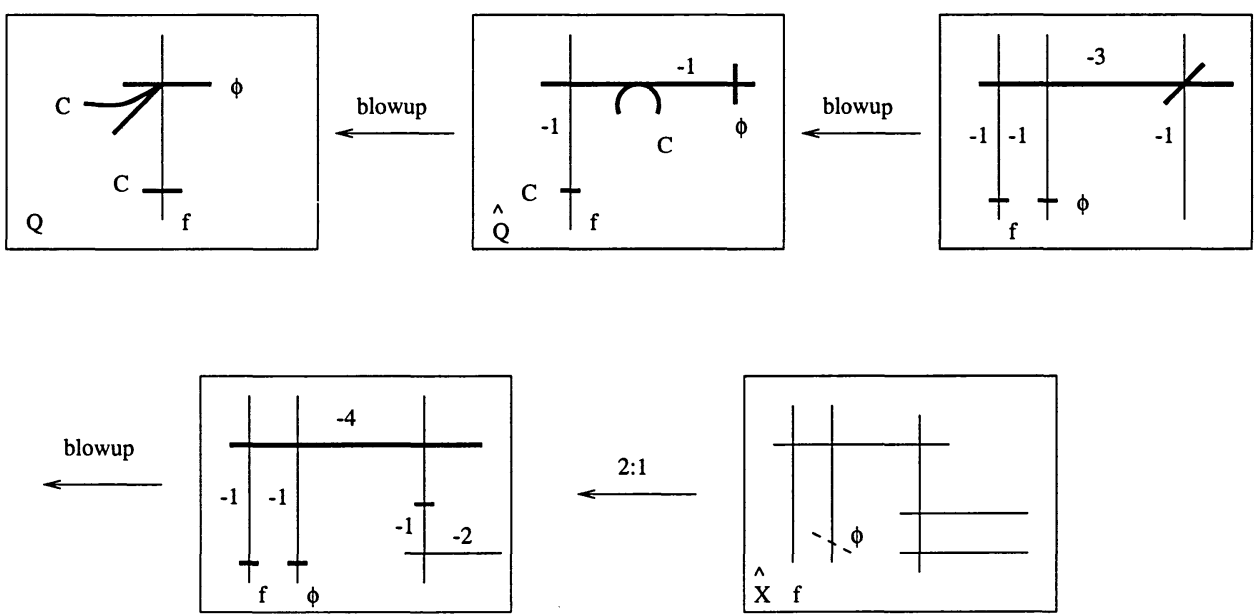

Figure 19: Collision leading to $S O(10)$ (inherited fibration). 

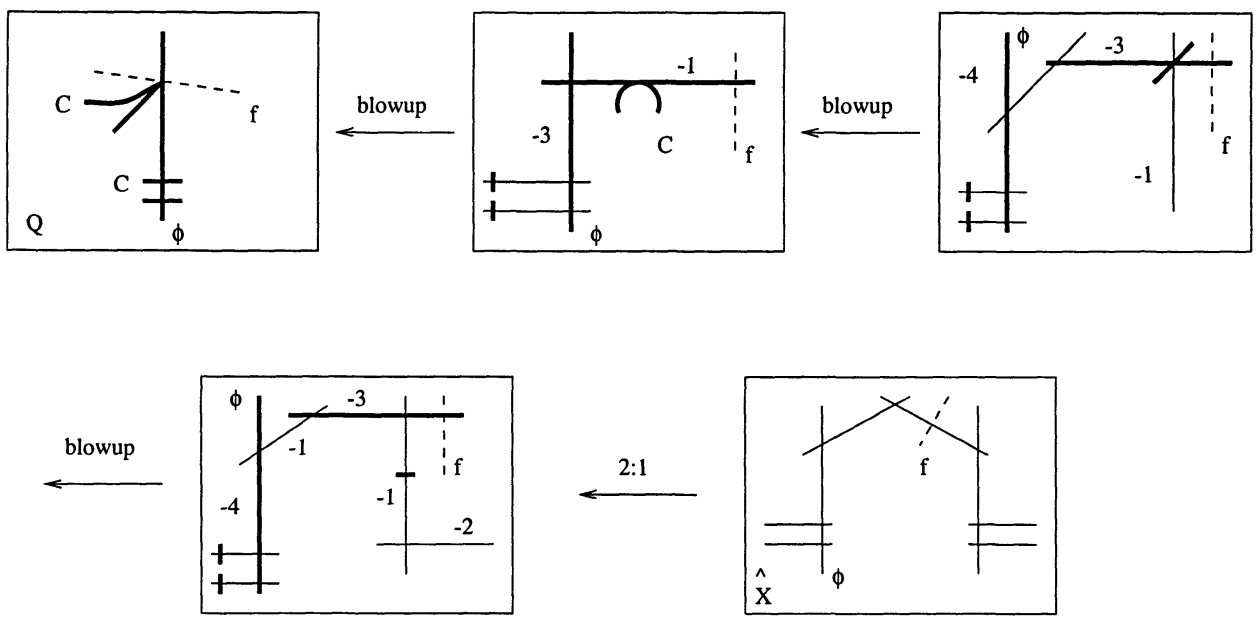

Figure 20: Collision leading to $S O(10)\left(\Gamma_{0}(2)\right.$ fibration).

and so the combined Dynkin diagram is

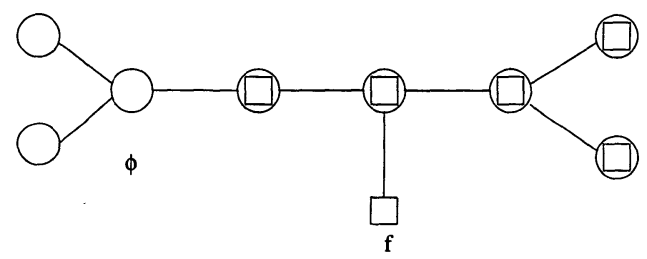

Figure 21: Combined Kodaira fibers producing $S O(10)$.

In general the $\Gamma_{0}(2)$ collision $2 I_{2}+n I_{1}$ with the two $I_{2}$ coming from different sections leads to a combined Dynkin diagram

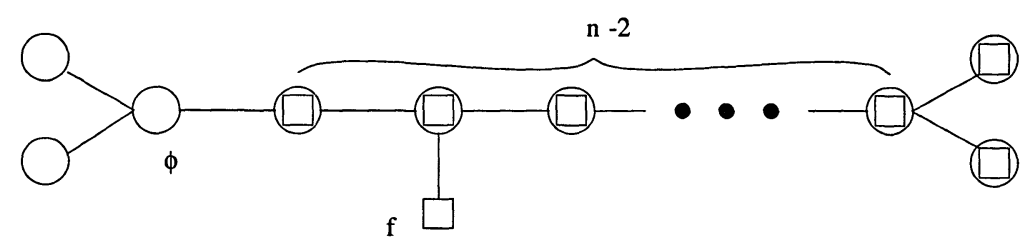

Figure 22: Combined Kodaira fibers producing $S O(2 n)$. and therefore leads to a gauge group $S O(2 n)$. 
Exceptional gauge groups $E_{6}, E_{7}, E_{8}$ : As in all of the cases above the appearance of the exceptional groups from the $E_{n}$ series will be governed by the degeneration of the branch curve $C \cup r_{1} \cup r_{2}$ to a curve with a singularity localized at a single fiber in either of the fibrations $q_{1}, q_{2}: Q \rightarrow \mathbf{P}^{1}$. Similarly to the $S O(2 n)$ case the degeneration of $C$ one needs to get the $E_{n}$ groups is special in the sense that the curve $C$ becomes globally reducible. More precisely a component of bidegree $(1,0)$ has to split of the $(2,4)$ curve $C$. Explicitly this means that the branch divisor of $X \rightarrow Q$ is of the form $r_{1} \cup r_{2} \cup r \cup C^{\prime}$ where $C^{\prime}$ is a curve of bidegree $(1,4)$ and $r$ is a $(1,0)$ curve. Observe that since $C^{\prime}$ is a numerical section of the fibration $q_{1}: Q \rightarrow \mathrm{P}^{1}$ we know that $C^{\prime}$ may be singular only if it is reducible.

In terms of the $\Gamma_{0}(2)$ fibration the reducibility of $C$ is equivalent to having a complete brake-up of the curve of 2-torsion points, i.e. of geting a monodromy group contained in $\Gamma(2) \subset \Gamma_{0}(2)$. In other words the $E_{n}$ gauge groups will come from collisions of fibers in the $\Gamma_{0}(2)$ fibration which occur in such a way that the monodromy group is kept contained in $\Gamma(2)$.

Gauge group $E_{6}$ : To obtain $E_{6}$ one needs a $\Gamma(2)$ collision of $6 I_{1}$. On the level of the branch curve this corresponds to having a smooth irreducible $C^{\prime}$ which is tangent to third order to $r_{3}$. The relevant blow-up pictures are shown in Figures 23, 24.
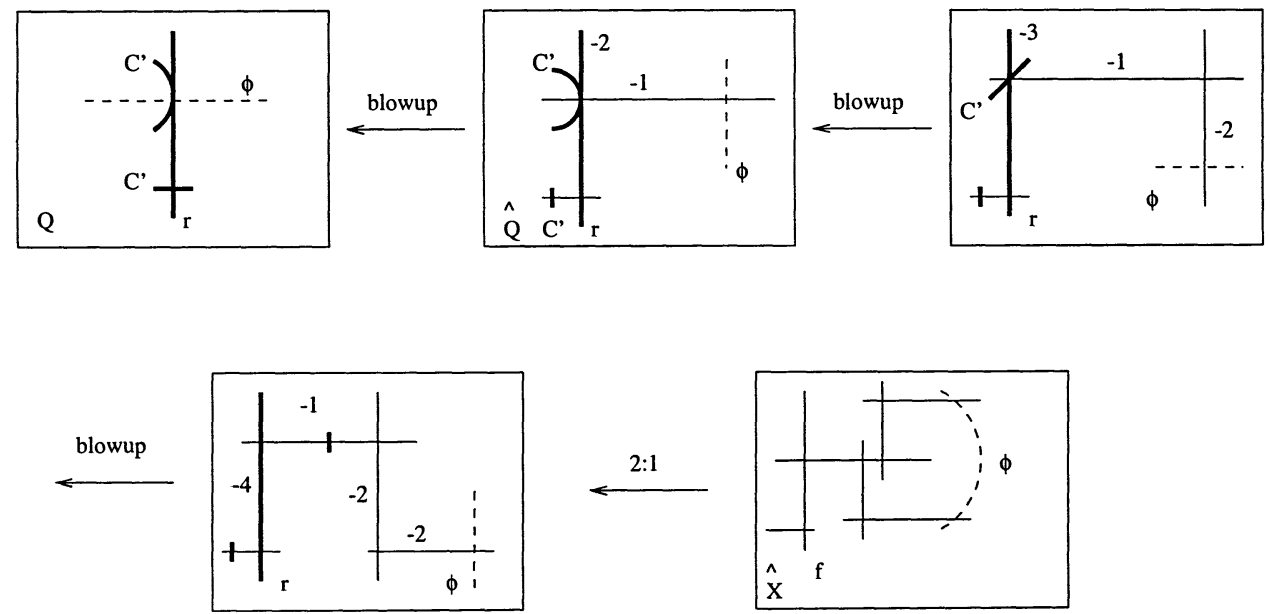

Figure 23: Collision leading to $E_{6}$ (inherited fibration). 

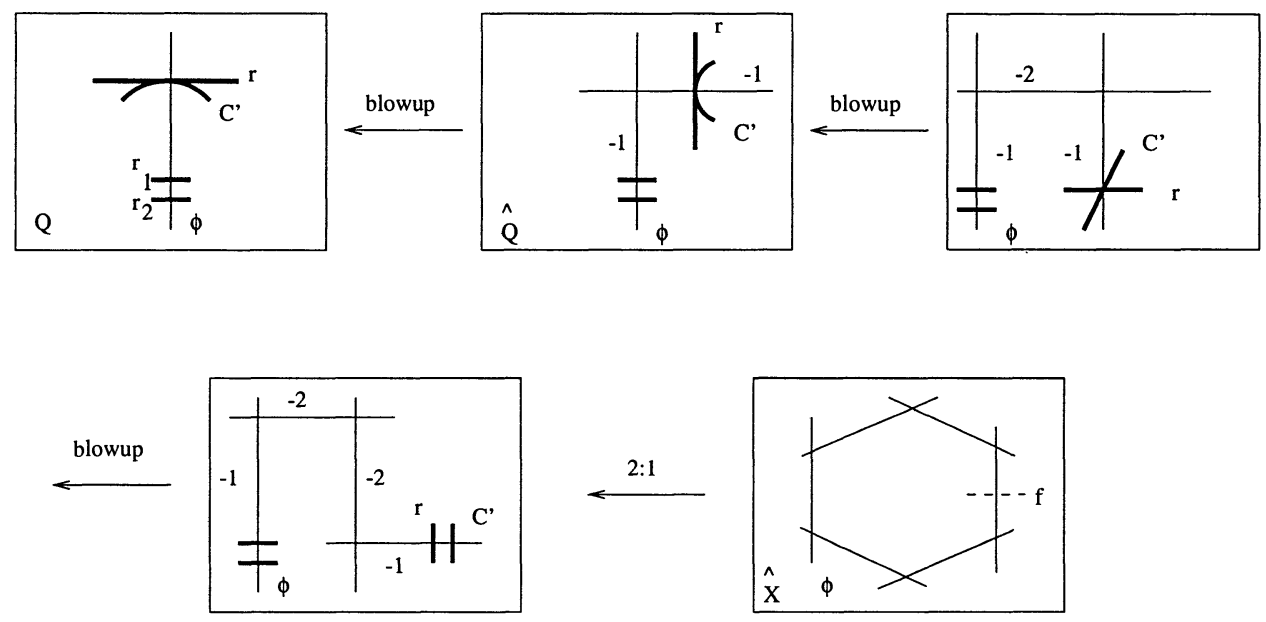

Figure 24: Collision leading to $E_{6}\left(\Gamma_{0}(2)\right.$ fibration).

where as usual $f$ denotes the preimage of the ruling $r$. The combined Dynkin diagram is:

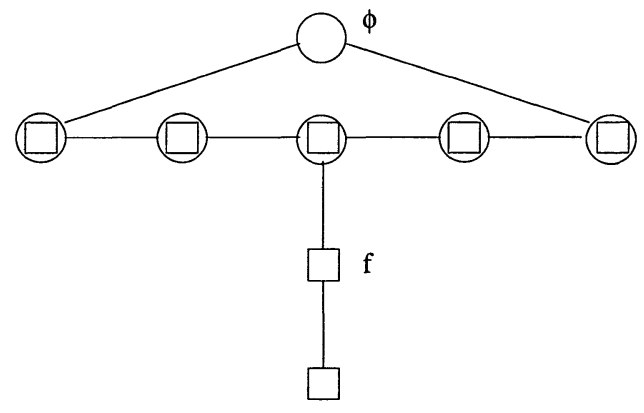

Figure 25: Combined Kodaira fibers producing $E_{6}$.

Note that in contrast with the cases with $A-C-D$ gauge symmetry the common part of the two Kodaira fibers does not equal the actual gauge group (which as before is just the complement of the affine root of the inherited fiber) but is only a part of it.

Gauge group $E_{7}: E_{7}$ can be obtained in two different ways trough $\Gamma(2)$ collisions. The first one which specializes to $E_{6}$ occurs when $C^{\prime}$ becomes tangent to fourth order to $r$, i.e. in the $\Gamma_{0}(2)$ fibration we have a $\Gamma(2)$ collision of $8 I_{1}$. The necessary blow-ups are shown in Figures 26, 27. 

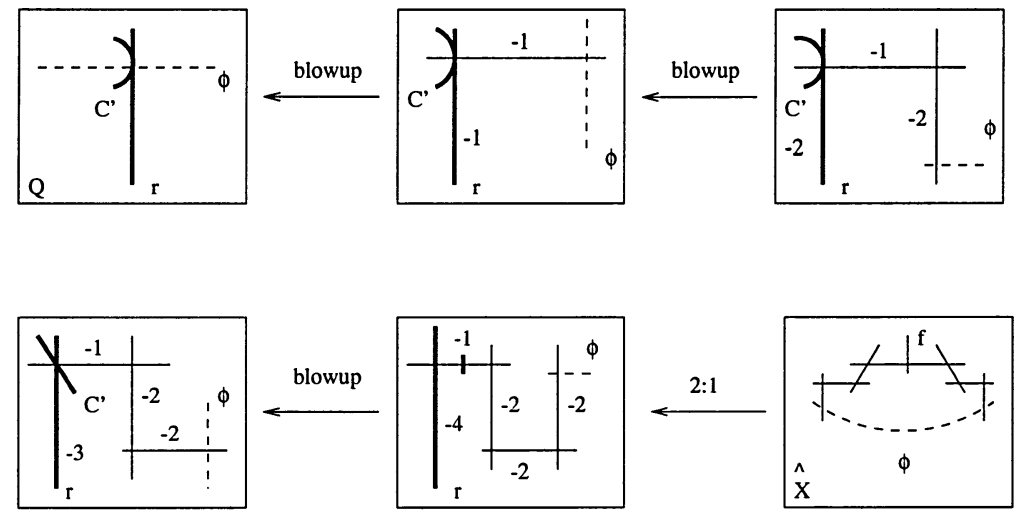

Figure 26: $\Gamma(2)$ collision of $8 I_{1}$ leading to $E_{7}$ (inherited fibration).
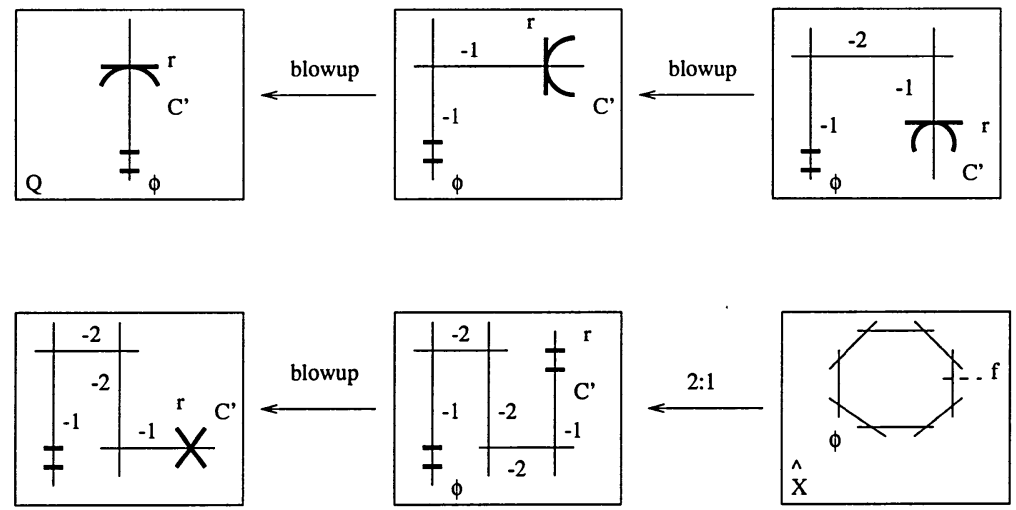

Figure 27: $\Gamma(2)$ collision of $8 I_{1}$ leading to $E_{7}$ ( $\Gamma_{0}(2)$ fibration).

where $f$ is again the preimage of the ruling $r$. The combined Dynkin diagram is:

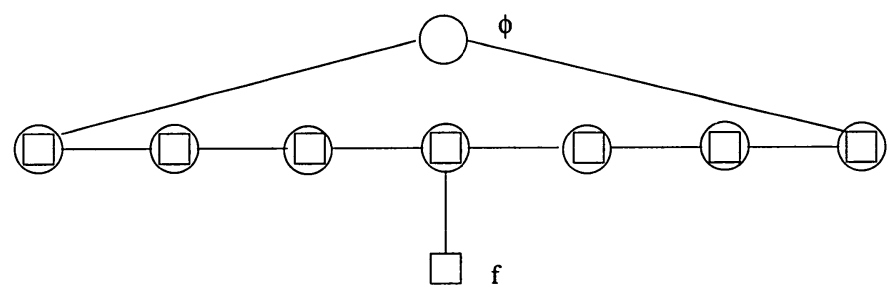

Figure 28: Combined Kodaira fibers producing $E_{7}$ in the $8 I_{1}$ collision.

The second $\Gamma(2)$ collision giving $E_{7}$ involves another global break- 
up. To obtain this collision one needs to find a degeneration of the configuration $r_{1} \cup r_{2} \cup r \cup C^{\prime}$ in which the curve $C^{\prime}$ becomes singular at a point of $r$. As observed above this can happen only if a component of bidegree $(0,1)$ splits of $C^{\prime}$. This component will necessarily hae to be a fiber of the projection $q_{2}$ and so we actually have a degeneration of the branch curve to a curve of the form $r_{1} \cup r_{2} \cup r \cup \phi \cup C^{\prime \prime}$ where as before $r_{1}, r_{2}, r$ are fibers for $q_{1}, \phi$ is a fiber of $q_{2}$ and $C^{\prime \prime}$ is a smooth irreducible curve of bidegree $(1,3)$. To get $E_{7}$ in this way the curve $C^{\prime \prime}$ has to be tangent to $r$ at the point of intersection of $r$ and $\phi$. In terms of the $\Gamma_{0}(2)$ fibration this corresponds to a $\Gamma(2)$ collision $2 I_{2}+6 I_{1}$ where the two $I_{2}$ 's come from different sections (or equivalently different groups of four in the Weierstrass model $Y$ ).

The blow-up sequence producing this $E_{7}$ fiber is shown in Figures 29,30 .
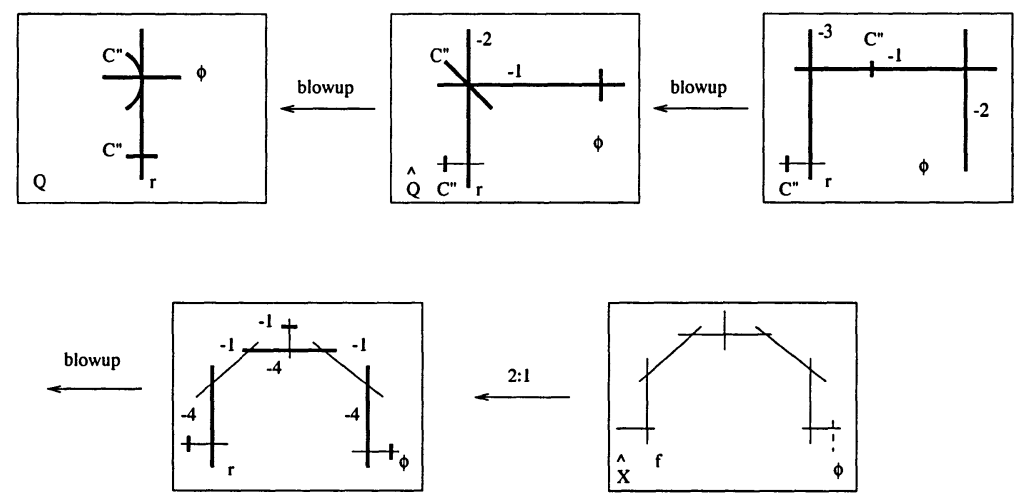

Figure 29: $\Gamma(2)$ collision of $2 I_{2}+6 I_{1}$ leading to $E_{7}$ (inherited fibration).
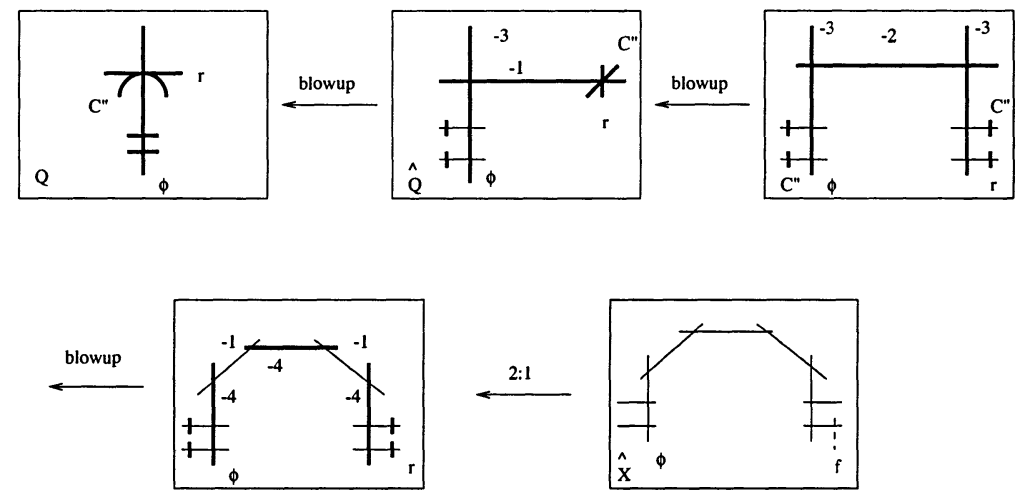

Figure 30: $\Gamma(2)$ collision of $2 I_{2}+6 I_{1}$ leading to $E_{7}$ ( $\Gamma_{0}(2)$ fibration). 
and the combined Dynkin diagram is:

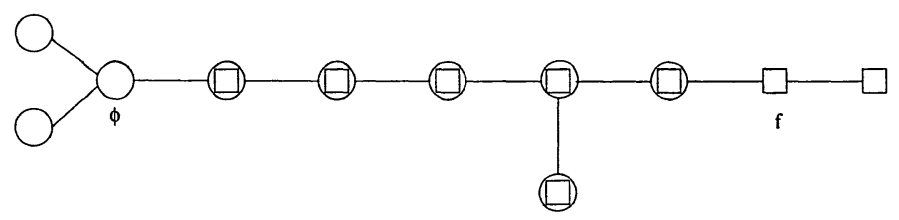

Figure 31: Combined Kodaira fibers producing $E_{7}$ in the $2 I_{2}+6 I_{1}$ collision.

Gauge group $E_{8}$ : The gauge group $E_{8}$ appears as a further degeneration of the $2 I_{2}+6 I_{1}$ producing $E_{7}$. More precisely the appearance of an $E_{8}$ fiber in the inherited fibration corresponds to a $\Gamma(2)$ collision of $2 I_{2}+8 I_{1}$ in the $\Gamma_{0}(2)$ fibration so that again the two $I_{2}$ come from different sections. The relevant blowup pictures are shown in Figures 32, 33.
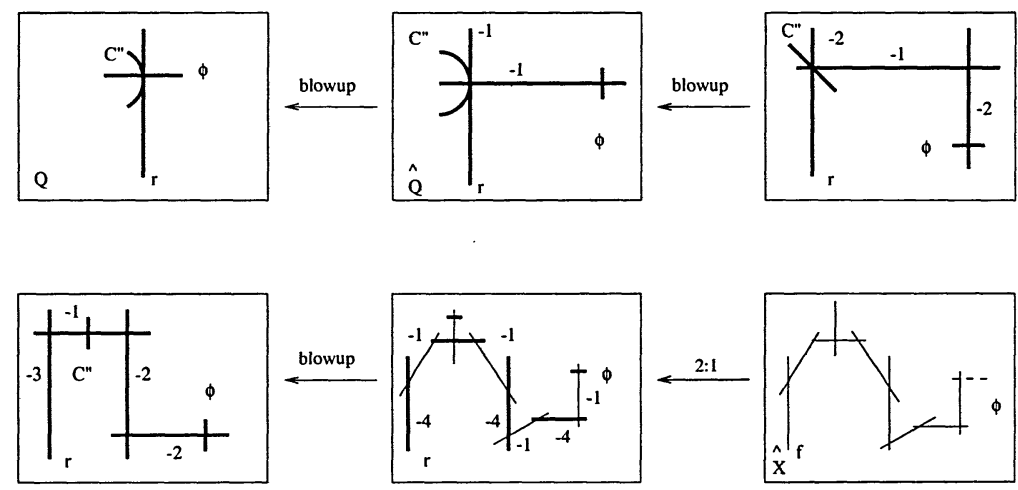

Figure 32: $\Gamma(2)$ collision of $2 I_{2}+8 I_{1}$ leading to $E_{8}$ (inherited fibration). 

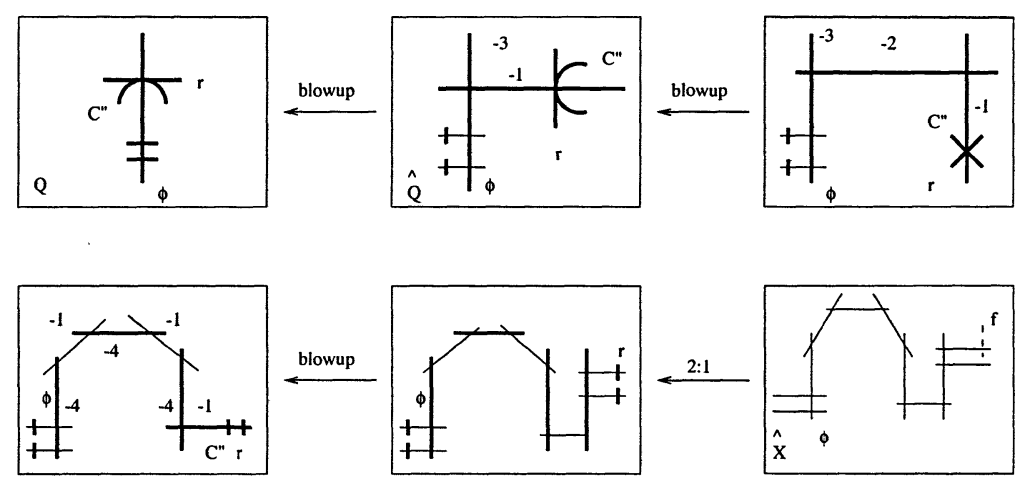

Figure 33: $\Gamma(2)$ collision of $2 I_{2}+8 I_{1}$ leading to $E_{8}$ ( $\Gamma_{0}(2)$ fibration).

and the combined Dynkin diagram is:

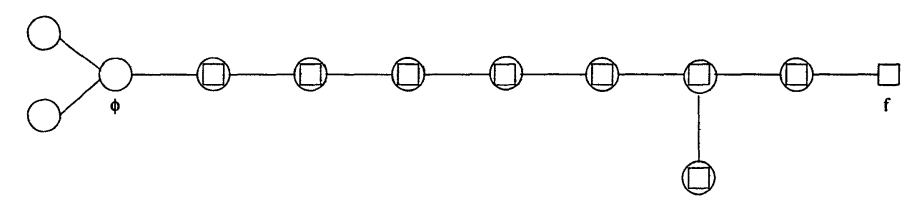

Figure 34: Combined Kodaira fibers producing $E_{8}$.

\section{A Appendix: Review of F-theory/Heterotic duality}

In this appendix we review the features of the F-theory-Heterotic string correspondence which are instrumental in recognizing the F-theory dual to a CHL string.

\section{A.1 The on-the-nose correspondence}

There are several ways in which one can justify this correspondence The model case is that of eight dimensional compactifications ( $\mathrm{cf}[21]$ ). In this case the heterotic string theory is compactified on a real two 
dimensional torus $T^{2}$ and is described by the following vacua ${ }^{20}$ : a complex structure $\tau$ on $T^{2}$, a complexified Kähler structure $\rho$ on $T^{2}$, as well as the Wilson lines of a gauge field on the torus with structure group $E_{8} \times E_{8}$ (or $\operatorname{Spin}(32) /(\mathbf{Z} / 2)$ ). The F-theory compactification on the other hand takes place on an elliptic K3 surface $X$ where the relevant fields ${ }^{21}$ are the complex structure on the K3 surface, the elliptic fibration structure $\pi: X \rightarrow \mathbf{P}^{1}$ on $X$ and a section $s: \mathbf{P}^{1} \rightarrow X$ of the elliptic fibration. The duality between the two theories is exhibited as an identification of their moduli spaces of vacua with the locally symmetric space

$$
O(2,18 ; \mathbf{Z}) \backslash O(2,18 ; \mathbf{R}) /(O(2 ; \mathbf{R}) \times O(18 ; \mathbf{R})) .
$$

For the heterotic string the identification of the moduli space with [?] is given by the Narain construction [23]. The identification for the F-theory moduli space is obtained through the global Torelli theorem for lattice polarized K3 surfaces $^{22}$. Concretely if $U$ denotes the two dimensional hyperbolic lattice and $\mathbf{E}_{8}$ denotes the root lattice of the group $E_{8}$ one considers the $\mathrm{K} 3$ lattice $\Lambda:=\mathrm{U}^{\oplus 3} \oplus \mathbf{E}_{8}^{\oplus 2} \cong \Gamma^{3,19}$. It is well known (see e.g. [4] ) that this lattice is (non-canonically) isomorphic to the second integral cohomology of any K3 surface. For any even non-degenerate lattice $M \subset \Lambda$ of signature $(1, \ell)$ a version of the global Torelli theorem (see the discussion after Proposition 3.3 in [11]) for K3 surfaces asserts that moduli space of $M$-polarized $\mathrm{K} 3$ surfaces is isomorphic to the space $\Gamma_{M} \backslash O\left(M^{\perp} \otimes \mathbf{R}\right) / K$. Here $K \subset O\left(M^{\perp} \otimes \mathbf{R}\right)$ is a maximal compact subgroup and $\Gamma_{M}=\operatorname{ker}\left[O\left(M^{\perp}\right) \rightarrow \operatorname{Aut}\left(\left(M^{\perp}\right)^{\vee} / M^{\perp}\right)\right]$. If $\pi: X \rightarrow \mathbf{P}^{1}$ is an elliptic $\mathrm{K} 3$ surface with a section the sublattice in $H^{2}(X, \mathbf{Z})$ generated by the class $f$ of the fiber and the class $s$ of the section is isomorphic to $\mathrm{U}$ (e.g. via the choice of basis $\{f, f+s\}$ ). By degenerating such a surface to an elliptic K3 surface with a section having two fibers of type $I I^{*}$ and four fibers of type $I_{1}$ one can easily see that the orthogonal complement of $\mathbf{Z} f \oplus \mathbf{Z} s$ is isomorphic to the lattice $\Gamma^{2,18}$ and hence if $\mathbf{U}$ is embedded in $\Lambda=\mathbf{U} \oplus \mathbf{U} \oplus \mathbf{U} \oplus \mathbf{E}_{8} \oplus \mathbf{E}_{8}$ as the first summand the moduli space of elliptic K3 surfaces with a section is

\footnotetext{
${ }^{20}$ In this discussion we have suppressed the heterotic string coupling constant

${ }^{21}$ The parameter corresponding to the heterotic string coupling appears in this context as the volume of the section of the elliptic fibration.

${ }^{22}$ Recall [11] that if $M$ is an even non-degenerate lattice of signature $(1, \ell)$ a K3 surface $S$ is called $M$-polarized if it is equipped with an inclusion $M \subset \operatorname{Pic}(S) \subset$ $H^{2}(S, \mathbf{Z})$.
} 
naturally identified with the moduli space of $U$-polarized K3 surfaces. On the other hand $U$ is self-dual and therefore $\Gamma_{U}=O\left(U^{\perp}\right)=O\left(\Gamma^{2,18}\right)$ which leads exactly to the locally symmetric space 10 .

\section{A.2 The correspondence in the stable limit}

The locally symmetric space 10 is in a natural way a quasi-projective algebraic variety due to the Bailey-Borel theorem. However the identifications with the F-theory and the heterotic string moduli just described are apriori only complex-analytic since they involve the period map for K3 surfaces and the complexified Kähler class $\rho$ respectively. However part of the identification is algebraic in nature which turns out to be crucial for the understanding of the six and four dimensional compactifications of the theories [5], [14], [12]. The algebraic geometry of the identifications above is best understood (see [21] and [14], section 4.4) in the limiting regime when the heterotic theory is decompactified by letting the complexified Kähler class on the two torus $T^{2}$ become infinitely large. This results in degeneration of the F-theory K3 to a K3 surface having two elliptic singularities of type $\tilde{E}_{8}$. The points obtained in this way on the boundary of the heterotic and F-theory moduli are at infinite distance and so do not correspond to vacua. On the other hand by understanding the local geometry of the moduli space near such points gives non-trivial information about the points in the interior.

Concretely, the surviving part of the heterotic vacua in the limit $|\rho| \rightarrow \infty$ is encoded in the algebraic-geometric data of an elliptic curve $Z$, which is just the torus $T^{2}$ considered as a complex manifold with the complex structure $\tau$, and an S-equivalence class $^{23}$ of semi-stable $E_{8} \times E_{8}$ principal bundle $V^{\prime} \times V^{\prime \prime} \rightarrow Z$. By letting the area of $Z$ go to infinity one effectively reduces the size of the instanton $V^{\prime} \times V^{\prime \prime}$ to zero which within the moduli space of $E_{8} \times E_{8}$ bundles on $Z$ can be viewed as a deformation of $V^{\prime} \times V^{\prime \prime}$ to the trivial bundle. As explained in [12] this deformation cannot be algebraic in general. On the other hand there is a natural algebraic deformation of the S-equivalence class of $V^{\prime} \times V^{\prime \prime}$ to the S-equivalence class of the trivial bundle. For this one just has to observe that the Looijenga moduli space of $E_{8}$ bundles on

\footnotetext{
${ }^{23}$ It is necessary to pass to $S$-equivalence since only the Wilson lines of the $E_{8} \times E_{8}$ instanton are relevant to the heterotic moduli.
} 
$Z$ is a weighted projective space and so there is a natural line passing trough every two points. In the following we will be working with this algebraic family of bundles on $Z$ rather than the family obtained from letting $|\rho| \rightarrow \infty$. This is justified by the expectation that there should be a global analytic automorphism of a tubular neighborhood of [23] that sends the two degenerations into each other. It will be very interesting to construct such an automorphism explicitly. From the Ftheory point of view the deformation of an elliptic K3 $X$ with a section to a $\mathrm{K} 3$ surface with two elliptic singularities of type $\tilde{E}_{8}$ corresponding to a coordinate of the period going to infinity can again be replaced by an algebraic degeneration which just uses the Weierstrass equation of $X$ (see [21] and [14], Section 4.4). In other words by varying the coefficients of the Weierstrass equation we can find a complex threefold $\mathcal{X}$ which fibers $\mathcal{X} \rightarrow \Delta$ over the unit disk $\Delta=\{t \in \mathbf{C}|| t \mid<1\}$ so that all the fibers $\mathcal{X}_{t}, t \neq 0$ are smooth elliptic K3 surfaces with a section, the fiber $\mathcal{X}_{0}$ over the point $0 \in \Delta$ is an elliptic K3 with two $\tilde{E}_{8}$ singularities and there is a point $\delta \in \Delta$ with $\mathcal{X}_{\delta} \cong X$.

The algebraic-geometric description of the F-theory-Heterotic duality in the limit is now described in two steps. First one replaces (see [14], Section 4.4 and [2]) the singular K3 with two isolated $\tilde{E}_{8}$ singularities by its stable model which is a normal crossing variety consisting of two del Pezzo surfaces of type $E_{8}$ glued transversally along an elliptic curve. To achieve this one performs semi-stable reduction on the family $\mathcal{X} \rightarrow \Delta$, i.e. uses a sequence of blow-ups and blow-downs on $\mathcal{X}$ and possibly finite base changes on $\Delta$ in order to replace $\mathcal{X}_{0}$ by a normal crossing variety. In the case under consideration the stable reduction requirest only two blow-ups followed by a blow-down. First one blows-up the two singular points of $\mathcal{X}_{0}$ in the threefold $\mathcal{X}$. The two exceptional divisors $X^{\prime}$ and $X^{\prime \prime}$ are rational elliptic surfaces with a section $^{24}$ and the strict transform of $\mathcal{X}_{0}$ is a $\mathbf{P}^{1}$-bundle over a copy of the elliptic curve $Z$. By contracting this $\mathbf{P}^{1}$ bundle onto the curve $Z$ one obtains [2] a family of surfaces over $\Delta$ which is isomorphic to $\mathcal{X}$ outside of $0 \in \Delta$ and whose central fiber is isomorphic to the transversal gluing $X^{\prime} \amalg_{Z} X^{\prime \prime}$ of $X^{\prime}$ and $X^{\prime \prime}$ along $Z$. The rational elliptic surfaces $X^{\prime}$ and $X^{\prime \prime}$ are naturally elliptically fibered (by their anticanonical linear system) and $Z$ sits in each of them as a fiber of the elliptic fibration. Since $X^{\prime} \coprod_{Z} X^{\prime \prime}$ has also a section which is a union of two $\mathbf{P}^{1}$ (each of them

\footnotetext{
${ }^{24}$ Recall that a rational elliptic surface with a section is a surface obtained as the blow-up of $\mathbf{P}^{2}$ in 9 points which are the intersection points of two cubic curves.
} 
in turn a section of the elliptic fibrations of $X^{\prime}$ and $X^{\prime \prime}$ respectively) meeting at the origin of $Z$ we can blow down these sections to obtain ${ }^{25}$ two del Pezzo surfaces $R^{\prime}, R^{\prime \prime}$ of type $E_{8}$ glued along the elliptic curve $Z$ siting inside each of them as an anticanonical section.

The second step needed for the duality in the limit is to interpret ([14], Section 4.1) any pair $Z \subset R$ consisting of an $E_{8}$ del Pezzo surface $R$ and an elliptic curve $Z$ sitting inside $R$ as an anticanonical section, as an S-equivalence class of semistable $E_{8}$ bundles on $Z$. In a rough outline this interpretation follows from viewing an $E_{8}$ bundle $V \rightarrow Z$ as a homomorphism $i_{V}: \mathbf{E}_{8} \rightarrow \hat{Z}, i_{V}(\lambda):=V \times_{E_{8}} \lambda$ from the $E_{8}$ lattice $\mathbf{E}_{8}$ to the dual elliptic curve $\hat{Z}$. Similarly given the pair $Z \subset R$ one gets a homomorphism $\operatorname{Pic}(R) \rightarrow \operatorname{Pic}(Z), L \mapsto L_{\mid Z}$. It is well known that the primitive part $\left\{L \in \operatorname{Pic}(R) \mid L \cdot K_{R}=0\right\}$ of the Picard group of an $E_{8}$ del Pezzo is isomorphic to the lattice $\mathbf{E}_{8}$. On the other hand since $Z$ is a section in $K_{R}^{-1}$ it follows that for any $L \in \operatorname{Pic}(R)$ for which $L \cdot K_{R}=0$ we wil have $\operatorname{deg}\left(L_{\mid Z}\right)=0$. Thus the pair $Z \subset R$ can be viewed as a homomorphism $\mathbf{E}_{8} \rightarrow \hat{Z}$ as well.

\section{Acknowledgments}

We are grateful to Oren Bergman, Ron Donagi, Andrey Johansen, Zurab Kakushadze, and Cumrun Vafa for useful discussions. The research of M. B. and V. S. was partially supported by the NSF grant PHY-92-18167, the NSF 1994 NYI award and the DOE 1994 OJI award. The research of T.P. was supported in part by NSF grant DMS-9800790.

\section{References}

[1] P. Aspinwall, M-Theory Versus F-Theory Pictures of the Heterotic String, Adv. Theor. Math. Phys., 1 (1998), 127-147, hep-th/9707014.

[2] P. Aspinwall, D. Morrison, Point-like Instantons on K3 Orbifolds, Nucl.Phys., B503 (1997), 533-564, hep-th/9705104.

${ }^{25}$ Recall that an $E_{8}$ del Pezzo is just the blow up of $\mathbf{P}^{2}$ at eight points in general position. 
[3] W. Barth, C. Peters, Automorphisms of Enriques surfaces, Invent. Math., 73 (1983), 383-411.

[4] W. Barth, C. Peters, A. van de Ven, Compact complex surfaces, Ergebnisse der Mathematik und ihrer Grenzgebiete (3) 4, SpringerVerlag, 1984.

[5] M. Bershadsky, A. Johansen, T. Pantev, and V. Sadov, On FourDimensional Compactifications of F-Theory, Nucl.Phys., B505 (1997), 165-201, hep-th/9701165.

[6] M. Bianchi, A Note on Toroidal Compactifications of the Type I Superstrings and other Superstrings Vacuum Configurations with 16 Supercharges, hep-th/9711201.

[7] M. Bianchi, G. Pradisi, and A. Sagnotti, Nucl. Phys., B376 (1990), 365.

[8] S. Chaudhuri, J. Polchinski, Moduli space of CHL strings, Phys.Rev., D52 (1995), 7168, hep-th/9506048.

[9] A. Connes, M. Douglas, and A. Schwarz, Non commutative Geometry and Matrix Theory: Compactification on a Tori, hep-th/9711162.

[10] F.Cossec, I.Dolgachev, Enriques surfaces I, Progress in Math., Vol. 76, Birkhäuser, 1989.

[11] I. Dolgachev, Mirror symmetry for lattice polarized K3 surfaces, alg-geom/9502005.

[12] R. Donagi, ICMP lecture on Heterotic/F-theory duality, hep-th/9802093.

[13] M. Douglas and C. Hull, D-branes and the Noncommutative Torus, hep-th/9711165.

[14] R. Friedman, J. Morgan, E. Witten, Vector Bundles And F Theory, Commun.Math.Phys., 187 (1997), 679-743, hep-th/9701162.

[15] P. Griffiths, Infinitesimal variations of Hodge structures (III): determinantal varieties and the infinitesimal invariant of normal functions, Comp. Math., 50 (1983) 267-324. 
[16] P. Griffiths, J. Harris, Principles of algebraic geometry, WileyInterscience, New York, 1978.

[17] Shamit Kachru, Albrecht Klemm, and Yaron Oz, Calabi-Yau Duals for CHL Strings, hep-th/9712035.

[18] K. Kodaira, Compact analytic surfaces III, Ann. of Math.

[19] W. Lerche, C. Schweigert, R. Minasian, and S. Theisen, A Note on the Geometryof CHL Heterotic String, hep-th/9711104.

[20] D. Morrison and C. Vafa, Compactifications of F-theory on CalabiYau threefolds-I, II, Nucl. Phys., B 473 (1996), 74; ibid, B 476 (1996), 437.

[21] D. Morrison, C. Vafa, Compactifications of F-Theory on Calabi-Yau Threefolds - II, Nucl.Phys., B476 (1996), 437-469, hep-th/9603161.

[22] Y. Namikawa, Periods of Enriques surfaces, Math. Annalen, 270 (1985), 201-222.

[23] K.S. Narain, New heterotic string theories in uncompactified dimensions less than 10, Phys. Let., 169B (1986), 41-46.

[24] J. Park, Phys. Lett., B418 (1998), 91.

[25] A.Sen, Nucl. Phys., B475 (1996), 562.

[26] A. Sen, J. Schwarz, Type IIA Dual of the Six-Dimensional CHL Compactification, Phys. Lett., B357 (1995), 323, hep-th/9507027.

[27] E. Witten, Toroidal Compactification Without Vector Structure, hep-th/9712028. 